\title{
The Role of Circulating Lycopene in Low-Grade Chronic Inflammation: A Systematic Review of the Literature
}

\author{
Hidde P. van Steenwijk ${ }^{1, *(\mathbb{D})}$, Aalt Bast ${ }^{2,3}\left(\mathbb{D}\right.$ and Alie de Boer ${ }^{1}(\mathbb{D}$ \\ 1 Campus Venlo, Food Claims Centre Venlo, Faculty of Science and Engineering, Maastricht University, \\ 5911 BV Venlo, The Netherlands; a.deboer@maastrichtuniversity.nl \\ 2 Campus Venlo, University College Venlo, Maastricht University, 5911 BV Venlo, The Netherlands; \\ a.bast@maastrichtuniversity.nl \\ 3 Department of Pharmacology \& Toxicology, Medicine and Life Sciences, Faculty of Health, \\ Maastricht University, 5911 BV Venlo, The Netherlands \\ * Correspondence: h.vansteenwijk@maastrichtuniversity.nl; Tel.: +4-3388-3666
}

Academic Editors: Rafael Guillén Bejarano and Rocío Rodríguez Arcos María Received: 20 August 2020; Accepted: 21 September 2020; Published: 23 September 2020

\begin{abstract}
Background and aims: In recent years, it has become clear that low-grade chronic inflammation is involved in the onset and progression of many non-communicable diseases. Many studies have investigated the association between inflammation and lycopene, however, results have been inconsistent. This systematic review aims to determine the impact of circulating lycopene on inflammation and to investigate the effect of consuming tomato products and/or lycopene supplements on markers of inflammation. Methods: Eligible studies, published before March 2020, were identified from PubMed, EBSCOhost and ScienceDirect. Human studies published in English, that evaluated the effect of circulating lycopene in relation to inflammation biomarkers were screened and included. Studies assessing lycopene intake or general intake of carotenoids/antioxidants without measuring circulating lycopene, as well as those not reporting inflammation biomarkers as outcomes, were excluded. Results: Out of 80 publications identified and screened, 35 met the inclusion criteria. Results from 18 cross-sectional studies suggest that lycopene levels are adversely affected during inflammation and homeostatic imbalance. Most of the 17 included intervention studies reported increased circulating lycopene levels after tomato/lycopene supplementation, but almost no changes in inflammation biomarkers were observed. Conclusions: There is little evidence that increasing tomato intake or lycopene supplementation diminuates this inflammation. However, depletion of lycopene may be one of the first signs of low-grade inflammation. The available data thereby imply that it is beneficial to consume lycopene-rich foods occasionally to stay healthy and keep circulating lycopene at a basal level.
\end{abstract}

Keywords: carotenoids; phytochemicals; bioactive; nutrition; antioxidant paradox

\section{Introduction}

The understanding of health has changed in recent years: in addition to medicine and pharmacology, there has been an increasing interest in lifestyle medicine in which nutrition plays a pivotal role [1]. In addition to conventional drug therapies, lifestyle adjustments, such as dietary changes, are also advised to reduce disease. Diets with a high proportion of fruits and vegetables seem to have a particularly positive effect on nutritional status as well as different non-communicable diseases, such as heart diseases, neurodegenerative diseases, and diabetes type II. As most non-communicable diseases are partially affected by inflammation, more research is being conducted on potential anti-inflammatory substances derived from fruits and vegetables [2-6]. 


\subsection{Low-Grade Chronic Inflammation}

Previous research has shown that the onset and progression of many non-communicable diseases, including heart diseases, neurodegenerative diseases, and diabetes type II, are (partly) related to, or affected by inflammation: low-grade chronic inflammation is central to many different symptoms from which patients suffer in these conditions. Chronic inflammation is believed to aggravate various mechanisms that reflect poor health, including elevated blood pressure, high blood sugar, excessive waist circumference, and abnormal cholesterol or triglyceride levels (the so-called "deadly quartet") [7]. In normal homeostasis, the function of inflammation is to eliminate the initial cause of cell injury, dispose of necrotic cells and damaged tissue caused by both the injury and the inflammation, and to initiate tissue repair. This natural response, acute inflammation, is a critical survival mechanism used by all higher vertebrates [8]. However, if acute inflammation is not resolved, it can lead to chronic inflammation, which is not part of the body's natural healing process and can constitute a damaging process. Damaged tissues release pro-inflammatory cytokines and other biological inflammatory mediators into the circulation, converting tissue-based low-grade inflammation into a systemic inflammatory condition. Moreover, autoimmune disorders and long-term exposure to irritants can also lead to a systemic inflammatory condition [8-10].

The inflammatory response is the coordinated activation of signaling pathways that regulate inflammatory mediator levels in resident tissue cells and inflammatory cells recruited from the blood. Although inflammatory response processes depend on the precise nature of the initial stimulus and its location in the body, for example, bacterial pathogens trigger Toll-like receptors (TLRs) and viral infections trigger type I interferons (IFN), they all share a common mechanism, which can be summarized as follows: (1) Cell surface pattern receptors recognize detrimental stimuli; (2) inflammatory pathways are activated; (3) inflammatory markers are released; and (4) inflammatory cells are recruited $[9,11]$. Inflammatory stimuli activate intracellular signaling pathways that subsequently activate the production of inflammatory mediators. Primary inflammatory stimuli, including microbial products and cytokines such as interleukin-1 $\beta$ (IL-1 $\beta$ ), interleukin-6 (IL-6), and tumor necrosis factor- $\alpha$ (TNF- $\alpha$ ), mediate inflammation through interaction with the TLRs, IL-1 receptor (IL-1R), IL-6 receptor (IL-6R), and the TNF receptor (TNFR). This receptor activation triggers important intracellular signaling pathways, including the mitogen-activated protein kinase (MAPK), nuclear factor kappa-B (NF-KB), NF-E2 p45-related factor 2 (Nrf2), and Janus kinase (JAK)- signal transducer, and activator of transcription (STAT) pathways [11]. In the state of low-grade chronic inflammation, a typical inflammatory stimulator or pathogen can no longer be determined, and inflammatory stimuli and pathways remain activated. Inflammatory stimuli, such as IL-6 and C-reactive protein (CRP), can then be used as biomarkers to measure inflammation [12].

Low grade inflammation is involved in the progression of many non-communicable diseases, but also seems to affect apparently healthy people as a consequence of smoking, stress, or alcohol consumption [8]. A wealth of epidemiological evidence indicates that overall health is strongly influenced by diets with a high proportion of fruits and vegetables $[2-4,13,14]$. Phytochemicals with anti-inflammatory activity present in fruits and vegetables are believed to be largely responsible for overall health. Therefore, new possibilities may exist in the reduction and prevention of non-communicable diseases by increasing the intake of anti-inflammatory food (ingredients) in both healthy and diseased individuals [15-17].

\subsection{Lycopene}

One group of nutritional compounds that has been suggested to elicit anti-inflammatory effects are carotenoids. As carotenoids are pigments in photosynthetic tissue, they are ubiquitous in leafy green vegetables. In non-photosynthetic tissue, carotenoids are responsible for the characteristic coloration of fruits such as red tomatoes, orange carrots, and red flesh in watermelon [18,19]. Of all carotenoids, a substantial amount of research has been conducted on the acyclic lycopene, present in e.g.; tomatoes. 


\subsubsection{Physicochemical Properties of Lycopene}

Lycopene has a chemical formula of $\mathrm{C} 40 \mathrm{H} 56$ and like all carotenoids, is a tetraterpene; assembled from eight isoprene units that are solely composed of hydrogen and carbon [20]. Lycopene is an acyclic isomer of $\beta$-carotene, however, unlike $\beta$-carotene lycopene lacks the $\beta$-ionic ring structure. Therefore, it lacks provitamin A activity $[20,21]$. However, lycopene is one of the most potent antioxidants, with a singlet-oxygen-quenching ability twice as high as that of $\beta$-carotene and ten times higher than that of $\alpha$-tocopherol (Vitamin E) [22]. Lycopene is a highly unsaturated, open-chain hydrocarbon containing eleven conjugated and two non-conjugated double bonds arranged in a linear array. The double bonds in lycopene can undergo isomerization from trans to cis isomers by thermal energy, chemical reactions, and light $[20,21]$. The all-trans isomeric form is primarily present in nature, followed by the 5-cis, 9-cis, 13-cis, and 15-cis isomeric forms. Several methods for analysis of circulating lycopene are described. Methods differ in that (i) either plasma or serum lycopene is measured, (ii) multiple isomers, trans-lycopene or total lycopene are measured, (iii) circulating lycopene is adjusted for total cholesterol. The correction for total cholesterol has been made in more recent intervention studies because there is a risk of carotenoid status being misinterpreted in subjects on cholesterol-lowering therapy if they rely on crude serum or plasma levels.

\subsubsection{Lycopene Kinetics after Oral Administration: Absorption, Distribution, Metabolism, Excretion}

Absorption of lycopene is similar to that of other lipid soluble compounds. Ingested lycopene is incorporated into dietary lipid micelles and absorbed across the gastrointestinal tract via passive diffusion into the intestinal mucosal lining. Then they are incorporated into chylomicrons and released into the lymphatic system for transport to the liver. Lycopene is transported by lipoproteins in the blood for distribution to the different organs [23]. Because of its lipophilic nature, the primary carrier of lycopene is LDL and not HDL [24]. Generally, 10-30\% of dietary lycopene is absorbed with the remainder being excreted. The bioavailability of lycopene is greater from tomato paste than from fresh tomatoes. The increased absorption of lycopene from processed products is attributed to the presence of cis isomeric forms [25]. The absorption of lycopene in humans is influenced by several biological and lifestyle factors including gender, age, body mass index and composition, hormonal status, blood lipids concentrations, alcohol consumption, smoking, and the presence of other carotenoids in the consumed products [20]. When lycopene is administered as the all-trans isomer it rapidly isomerizes to a mixture containing more than $50 \%$ cis-isomers during absorption in the bloodstream and tissues. Moreover, a study showed that administration of all-trans lycopene in tomato sauce to human subjects for three weeks resulted in $77.3 \%$ cis isomers in prostate tissue and thus only $22.7 \%$ all-trans lycopene [26]. Liver, seminal vesicles, and prostate tissue are the primary sites of lycopene accumulation in humans [27]. Recent studies indicate that the accumulation in these sites may be due to the involvement of an active process for the uptake of carotenoids via the scavenger receptor class B type 1 protein (SR-B1) transporter, in addition to passive diffusion [28]. The full metabolic routes of lycopene in humans is still unclear. Only a few metabolites, such as 5,6-dihydroxy-5,6-dihydro-lycopene, have been detected in human plasma. It is suggested that lycopene may undergo in vivo oxidation to form epoxides which then may be converted to the polar 5,6-dihydoxy-5,6-dihydro-lycopene through metabolic reduction [29].

\subsubsection{Mechanism of Action (In Vitro)}

Lycopene has been shown to inhibit the binding abilities of NF-KB and stimulatory protein-1 (SP1), and decreased expression of insulin-like growth factor-1 receptor (IGF-1R) and intracellular ROS concentrations in human SK-Hep-1 cells [30]. Recently, Fenni et al. [31] confirmed the potential involvement of lycopene in decreasing the binding abilities of NF- $\mathrm{BB}$. They demonstrated the ability of lycopene supplementation to inhibit high-fat diet-induced obesity, inflammatory response, and associated metabolic disorder in mice. They evaluated the effect of lycopene on the phosphorylation 
of p65 and IкB, which are involved as modulators in the NF- $\mathrm{B}$ pathway. Lycopene was able to strongly reduce phosphorylation of p 65 and I $\mathrm{k} B$, resulting in the deactivation of the NF- $\kappa B$ pathway, that previously was induced by the consumption of a high-fat diet. This effect can thus be seen as the induction of an anti-inflammatory effect. These results have also been observed in SW480 human colorectal cancer cells, where lycopene restrained NF- $\mathrm{KB}$ and JNK activation, resulting in a suppression of TNF- $\alpha$, IL-1 $\beta$, IL-6, COX-2, and iNOS expression. However, relatively high concentrations of lycopene were used (10-30 $\mu \mathrm{M})$ compared to usual detectable plasma levels $(1-2 \mu \mathrm{M})$ [32].

While in vitro and animal studies show promise for the potential health effects of lycopene, the relationship between lycopene and low-grade chronic inflammation in itself has so far been inconclusive in humans. Various systematic reviews have already been conducted on lycopene and how it affects different diseases and their symptoms, such as prostate and bladder cancer, Cardiovascular risk and metabolic syndrome [33-36]. The cross-sectional and intervention studies assessed in these reviews were often inconclusive, and the inconsistency among studies and the type of lycopene tested makes comparison difficult. The different lycopene measurements (self-reported FFQ, measurement of product, circulating lycopene) are a possible reason for the inconsistent results. Circulating measures are preferred for assessing relations, because self-reported measures of lycopene intake are subject to recall bias or memory error and intake measurements do not provide insight in the absorption, distribution, metabolism, and excretion of lycopene in the body. For in vivo studies, however, it is necessary to not just focus on lycopene intake but to actually measure the circulating lycopene concentrations in plasma or serum, in order to understand the health effects on humans [21]. C-reactive protein (CRP) and interleukin-6 (IL-6) are most commonly used to measure inflammation, but some studies have reviewed other inflammatory biomarkers (hyaluronic acid (HA), malondialdehyde (MDA), adiponectin, monocyte chemoattractant protein 1 (MCP-1), thiobarbituric acid reactive substances (TBARS), serum amyloid A (SAA), tumor necrosis factor- $\alpha$ (TNF- $\alpha$ ), interleukin-1 $\beta$ (IL-1 $\beta)$ ). These will also be included in this study $[37,38]$. As such, this is the first systematic review of the literature to investigate the relationship between circulating lycopene and inflammation.

\section{Methods}

\subsection{Literature Search}

This systematic review was conducted following the Cochrane and the Centre for Reviews and Dissemination guidelines on systematic reviews and is reported according to PRISMA guidelines [39,40]. This systematic review of the literature was conducted to investigate the relationship between circulating lycopene and inflammation in order to understand the health effects of lycopene in humans. To identify relevant human studies in which the relationship between lycopene and inflammatory markers was measured, a systematic search was conducted in Pubmed, EBSCOhost and ScienceDirect as databases. Using the Boolean search terms "serum lycopene" and "inflammation," articles published in peer-reviewed journals in the English language were flagged for further review. As this review focuses on the effect of lycopene intake on inflammation in vivo, only human studies were considered for inclusion. The date of publication did not serve as an exclusion criterion. The search was conducted in duplicate by the first and last author and all potentially relevant publications up to March 2020 were included in the search.

The following search terms yielded 42 articles in Pubmed and 13 articles in EBSCOhost: (("serum"[MeSH Terms] OR "serum"[All Fields]) AND ("lycopene"[MeSH Terms] OR "lycopene"[All Fields]) AND ("inflammation"[MeSH Terms] OR "inflammation"[All Fields])).

In ScienceDirect, the search terms "lycopene" and "inflammation" in "Find articles with these terms" and in "Title, abstract or author-specified keywords," 79 articles published in English from peer-reviewed journals were flagged for further review in ScienceDirect. After removing duplicates (62), a total of 72 articles were therefore screened for inclusion in this systematic review. Additionally, the reference list of each included article was examined to identify any additional studies for inclusion 
that might not have appeared in the search results. This led to the identification of eight more articles for further review.

\subsection{Application of Inclusion/Exclusion Criteria}

As this systematic review focusses on circulating lycopene, only studies that report serum or plasma lycopene levels as independent measures and their relation to inflammation (inflammatory biomarkers) were considered for inclusion in this review. Although human studies were selected in the search terms, the search still identified a few in vitro and animal studies, which were subsequently excluded (3). Only full text, original human research studies were included. This led to the exclusion of two additional studies that presented research only as an abstract or in the form of a presentation.

Various studies were seen to report merely the intake of lycopene and its relationship with inflammation biomarkers, whereas the main interest of this study is to identify actual levels of lycopene in plasma/serum detected after/following consumption. Therefore, when analyzing full text versions of all studies, studies were excluded from this assessment based on the following exclusion criteria: (i) Assessment of lycopene intake or general intake of carotenoids/antioxidants without measuring circulating lycopene; and (ii) no inflammation biomarkers reported as outcomes. In total, the screening and eligibility process of this literature search led to the identification of 35 studies that were included and subjected to critical analysis (Figure 1).

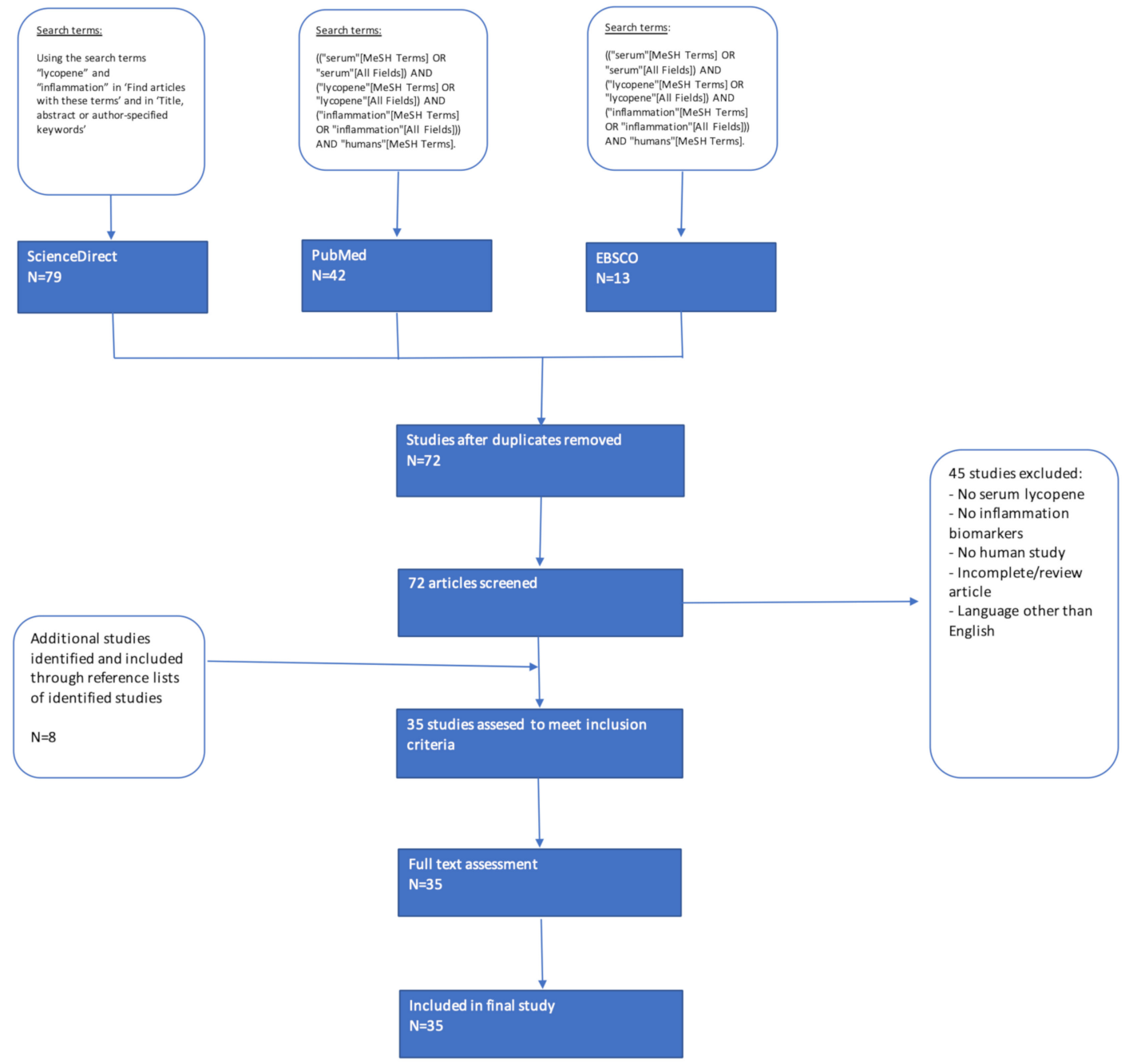

Figure 1. Flowchart of systematic search strategy. 


\subsection{Data Extraction and Analysis}

To assess the quality and to minimize the risk of reporting bias, each author independently analyzed the articles. The authors discussed the extracted data and thoroughly considered differing interpretations before establishing consensus. Extracted information included: study design, randomization, duration and length of follow-up, methods of analysis, participants characteristics (population, settings of intervention, baseline characteristics), outcome measures (biomarkers), intervention details (i.e.; tomato or lycopene), and conclusions. Brief descriptions and summaries of results for the final articles included in this review are presented in Table 1: Cross-sectional studies assessing the relation between circulating lycopene and inflammation; and Table 2: Intervention studies assessing the influence of lycopene on inflammation.

Table 1. Cross-sectional studies assessing the relation between circulating lycopene and inflammation.

\begin{tabular}{|c|c|c|c|c|c|}
\hline Study (Ref) & Study Population & Final $n$ & Lycopene Measurement & $\begin{array}{l}\text { Inflammation } \\
\text { Biomarkers }\end{array}$ & Conclusions \\
\hline Mazidi et al. [41] & $\begin{array}{l}\text { Participants divided in } \\
\text { quartiles depending on } \\
\text { CRP and Fibrinogen }\end{array}$ & $\begin{array}{l}\text { Q1: }(n=193) \\
\text { Q2: }(n=190) \\
\text { Q3: }(n=183) \\
\text { Q4: }(n=199)\end{array}$ & $\begin{array}{c}\text { Serum trans-Lycopene } \\
(\mu \mathrm{mol} / \mathrm{L}) \\
\text { Q1: } 0.431 \pm 0.007 \\
\text { Q2: } 0.425 \pm 0.007 \\
\text { Q3: } 0.421 \pm 0.005 \\
\text { Q4: } 0.387 \pm 0.009\end{array}$ & $\begin{array}{l}\text { CRP }(\mathrm{mg} / \mathrm{dL}) \\
\text { Q1: } 0.03 \pm 0.01 \\
\text { Q2: } 0.14 \pm 0.04 \\
\text { Q3: } 0.33 \pm 0.07 \\
\text { Q4: } 1.2 \pm 0.89\end{array}$ & $\begin{array}{c}\text { A higher trans-lycopene level for } \\
\text { each } \mu \mathrm{mol} / \mathrm{L} \text { correlated with } 0.067 \\
\mathrm{mg} / \mathrm{dL} \text { lower CRP and } 0.048 \\
\mathrm{mg} / \mathrm{dL} \text { Fibrinogen }\end{array}$ \\
\hline $\begin{array}{l}\text { Crespo-Sanjuán et al. } \\
\text { [42] }\end{array}$ & $\begin{array}{l}\text { Control subjects }(n=14) \\
\text { Patients with intestinal } \\
\text { polyps }(n=39) \\
\text { Patients with colorectal } \\
\text { adenocarcinoma }(C R C) \\
\qquad(n=128)\end{array}$ & $\begin{array}{c}\text { Control }(n=14) \\
\text { Patients }(n=167)\end{array}$ & $\begin{array}{c}\text { Plasma Lycopene }(\mu \mathrm{g} / \mathrm{L}) \\
\text { Control: } 194.33 \pm 66.17 \\
\text { Carc. in Situ: } \\
138.57 \pm 106.62 \\
\text { Cancer IV: } 100.42 \pm 71.20\end{array}$ & $\begin{array}{c}\text { Plasma CRP }(\mathrm{mg} / \mathrm{L}) \\
\text { Control: } 2.05 \pm 2.33 \\
\text { Carc. in Situ: } \\
\text { 13.93 } \pm 26.53 \\
\text { Cancer IV: } 41.83 \pm 62.01\end{array}$ & $\begin{array}{l}\text { Levels of lycopene were higher in } \\
\text { the control group and low in the } \\
\text { stage-IV group ( } p=0.03) \text {, and } \\
\text { were inversely correlated with } \\
\text { CRP }(p=0.005, \mathrm{R}=-0.215) . \text { We } \\
\text { found a consistent relationship } \\
\text { between high lycopene and } \\
\text { absence of atherosclerosis } \\
(p=0.002) .\end{array}$ \\
\hline Kim et al. [43] & $\begin{array}{l}\text { Healthy women (31-75 } \\
\text { yrs) classified into } \\
\text { tertiles according to } \\
\text { serum lycopene } \\
\text { concentration }(n=264)\end{array}$ & $\begin{array}{l}\text { T1 }(n=88) \\
\text { T2 }(n=88) \\
\text { T3 }(n=88)\end{array}$ & $\begin{array}{c}\text { Serum Lycopene } \\
\text { (mmol/L) } \\
\text { T1: } 0.029 \pm 0.000 \\
\text { T2: } 0.039 \pm 0.000 \\
\text { T3: } 0.052 \pm 0.001\end{array}$ & $\begin{array}{c}\text { hs-CRP }(\mathrm{mg} / \mathrm{dL}) \\
\text { T1: } 1.27 \pm 0.24 \\
\text { T2: Data not shown T3: } \\
0.80 \pm 0.25\end{array}$ & $\begin{array}{c}\text { Subjects in T3 showed lower } \\
\text { C-reactive protein (hs-CRP) }(0.80 \\
\pm 0.25 \mathrm{mg} / \mathrm{dL} \text { vs. } 1.27 \pm 0.24 \\
\mathrm{mg} / \mathrm{dL}, p=0.015), \text { compared with } \\
\text { those in T1. }\end{array}$ \\
\hline Riccioni et al. [44] & $\begin{array}{l}\text { Participants } \\
\text { asymptomatic with } \\
\text { respect to carotid artery } \\
\text { disease divided over } 3 \\
\text { groups based on Carotid } \\
\text { intima-media thickness } \\
\qquad(n=640)\end{array}$ & $\begin{array}{l}\text { C1 }(n=291) \\
\text { C2 }(n=232) \\
\text { C } 3(n=117)\end{array}$ & $\begin{array}{c}\text { Plasma Lycopene } \\
(\mu \mathrm{mol} / \mathrm{L}) \mathrm{C} 1: 0.82 \pm \\
0.33 \mathrm{C} 2: 0.33 \pm 0.63 \mathrm{C} 3: \\
0.34 \pm 0.21\end{array}$ & $\begin{array}{c}\text { CRP }(\mathrm{g} / \mathrm{dL}) \mathrm{C} 1: \\
2.90 \pm 1.30 \\
\text { C2: } 3.84 \pm 1.75 \\
\text { C3: } 4.86 \pm 2.20\end{array}$ & $\begin{array}{c}\text { Elevated CIMT was significantly } \\
\text { associated with having a low } \\
\text { concentration of all antioxidants } \\
\text { evaluated (vitamin A, vitamin E, } \\
\text { lycopene, and b-carotene) and a } \\
\text { higher concentration of } \\
\text { inflammatory factors including } \\
\text { serum uric acid, CRP, } \\
\text { and fibrinogen. }\end{array}$ \\
\hline Hozawa et al. [45] & $\begin{array}{l}\text { Men and women in the } \\
\text { Coronary Artery Risk } \\
\text { Development in Young } \\
\text { Adults study (18-30 } \\
\text { years) divided in } \\
\text { quartiles depending on } \\
\text { Lycopene levels } \\
(n=4580)\end{array}$ & $\begin{array}{l}\text { Q1: }(n=1144) \\
\text { Q2: }(n=1144) \\
\text { Q3: }(n=1144) \\
\text { Q4: }(n=1148)\end{array}$ & $\begin{array}{l}\text { Serum Lycopene } \\
\text { (nmol/L) } \\
\text { Q1: } 24.2 \\
\text { Q2: } 44.1 \\
\text { Q3: } 62.0 \\
\text { Q4: } 91.8\end{array}$ & $\begin{array}{l}\text { CRP }(\mathrm{mg} / \mathrm{L}) \mathrm{Q} 1: 1.04 \mathrm{Q} 2: \\
\text { 1.11Q3: 0.99Q4: } 1.11\end{array}$ & $\begin{array}{l}\text { Serum total and individual } \\
\text { carotenoids, with the exception of } \\
\text { lycopene, were inversely } \\
\text { associated with markers of } \\
\text { inflammation }\end{array}$ \\
\hline Walston et al. [46] & $\begin{array}{l}\text { Subjects were disabled } \\
\text { women aged }>65 \text { years } \\
\qquad(n=619)\end{array}$ & $(n=619)$ & $\begin{array}{l}\text { Serum Lycopene } \\
\quad(\mu \mathrm{mol} / \mathrm{L}) \\
0.56 \pm 0.31\end{array}$ & $\begin{array}{l}\mathrm{IL}-6(\mathrm{pg} / \mathrm{mL}) \\
5.51 \pm 12.69\end{array}$ & $\begin{array}{l}\text { Persons with the highest levels of } \\
\text { b-carotene, lycopene, } \\
\text { lutein/zeaxanthin, } \\
\text { b-cryptoxanthin, and retinol were } \\
\text { also significantly less likely to be } \\
\text { in the highest interleukin- } 6 \text { tertile. }\end{array}$ \\
\hline Eboumbou et al. [47] & $\begin{array}{l}\text { Sudanese subjects } \\
\text { exposed and not } \\
\text { exposed to Schistosoma } \\
\text { infection and French } \\
\text { control subjects }\end{array}$ & $\begin{array}{l}\text { Rural Sudan: }(n=35) \\
\text { Urban Sudan: } \\
\quad(n=27) \\
\text { French: }(n=34)\end{array}$ & $\begin{array}{c}\text { Serum Lycopene } \\
(\mu \mathrm{M}) / \text { Lycopene:B-carotene } \\
\text { ratio } \\
\text { RS: } 0.21(0.04) / 1.10 \\
\text { US: } 0.68(0.10) / 5.11 \\
\text { F: } 1.10(0.25) / 4.52\end{array}$ & $\begin{array}{c}\text { Hyaluronic acid } \\
\text { (HA)/Malondialdehyde } \\
(\mathrm{MDA}) \\
\text { around } 60 \mu \mathrm{g} / \mathrm{L} / 200 \mathrm{nM}\end{array}$ & $\begin{array}{l}\text { Drastic decrease of lycopene } \\
\text { levels in the subjects exposed to } \\
\text { schistosomiasis in comparison } \\
\text { with non-exposed Sudanese and } \\
\text { French control subjects }\end{array}$ \\
\hline $\begin{array}{l}\text { van Herpen-Broekmans } \\
\text { et al. [48] }\end{array}$ & $\begin{array}{l}\text { Healthy men and } \\
\text { women }(n=379)\end{array}$ & $\begin{array}{l}\text { Men: }(n=178) \\
\text { Women: }(n=201) \\
\text { Total: }(n=379)\end{array}$ & $\begin{array}{c}\text { Serum Lycopene } \\
(\mu \mathrm{mol} / \mathrm{L}) \\
\text { Men: } 0.35 \pm 0.18 \\
\text { Women: } 0.37 \pm 0.18 \\
\text { Total: } 0.36 \pm 0.18\end{array}$ & $\begin{array}{c}\text { CRP }(\mathrm{mg} / \mathrm{L}) \\
\text { Men: } 0.9(0.2-5.9) \\
\text { Women: } 1.4(0.2-7) \\
\text { Total: } 1.1(0.2-6.7)\end{array}$ & $\begin{array}{c}\text { An inverse relation between } \\
\text { lycopene and CRP }(-1.14 \pm 0.54 \\
\text { per umol/l; } p=0.04) \text { was found in } \\
\text { men and not in women }(0.50 \pm \\
0.50 \text { per umol/l; } p=0.32)\end{array}$ \\
\hline
\end{tabular}


Table 1. Cont.

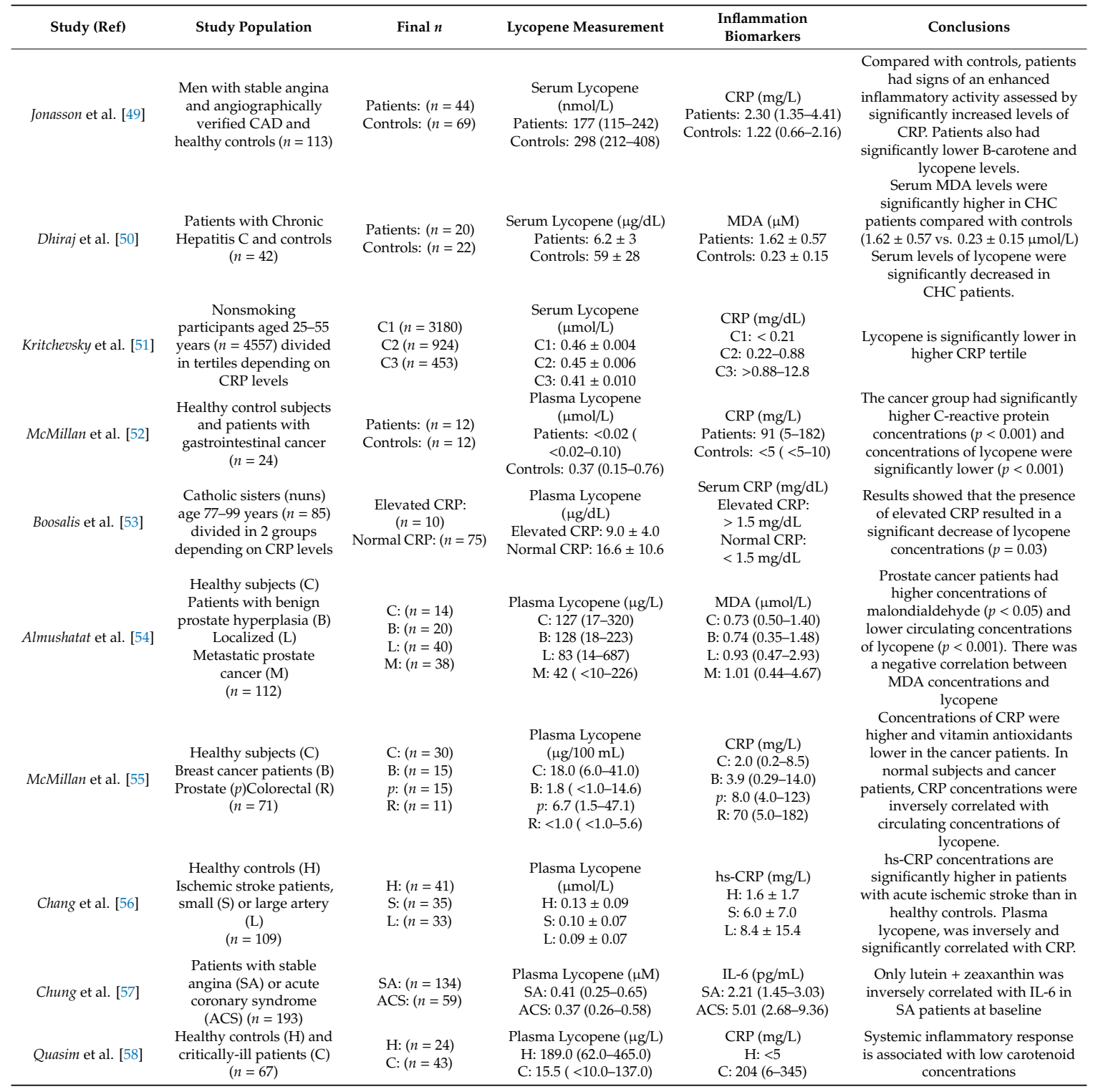


Table 2. Intervention studies assessing the influence of lycopene supplementation on inflammation.

\begin{tabular}{|c|c|c|c|c|c|c|}
\hline Study (Ref) & Study Population & Intervention & Final $n$ & Lycopene Measurement & Inflammation Biomarkers & Conclusions \\
\hline Nieman et al. [59] & Healthy runners $(n=20)$ & $\begin{array}{l}\text { Lycopene capsule }(5 \mathrm{mg} / \mathrm{d}) \text { or } \\
\text { placebo } \\
\text { for } 4 \text { weeks }\end{array}$ & $(n=20)$ & $\begin{array}{l}\text { Plasma Lycopene }(\mathrm{ng} / \mathrm{mL}) \\
\text { Pre-supplement: around } 500 \\
\text { Post-supplement: around } 750\end{array}$ & $\begin{array}{c}\text { CRP }(\mathrm{mg} / \mathrm{L}) \\
\text { Pre-supplement: } 1.21 \pm 1.2 \\
\text { Post-supplement: } 1.28 \pm 1.0\end{array}$ & $\begin{array}{l}\text { Plasma lycopene increased } \\
\text { significantly in intervention group } \\
\text { compared to placebo }(p<0.000) \text {. No } \\
\text { alterations in post-exercise measures } \\
\text { of oxidative stress and inflammation } \\
\text { were found. }\end{array}$ \\
\hline$L i$ et al. [60] & $\begin{array}{l}\text { Healthy young Taiwanese } \\
\text { females }(n=25)\end{array}$ & $\begin{array}{c}100 \% \text { pure tomato juice, } \\
\text { containing } 11.6 \mathrm{mg} \text { of lycopene } \\
\text { per } 100 \mathrm{~mL} \\
280 \mathrm{~mL} / \text { day for } 56 \text { days }\end{array}$ & $(n=25)$ & $\begin{array}{c}\text { Serum Lycopene }(\mu \mathrm{M}) \\
\text { Pre-supplement: } 0.72 \pm 0.36 \\
\text { Post-supplement: } 1.94 \pm 0.74\end{array}$ & $\begin{array}{c}\text { Adiponectin }(\mu \mathrm{g} / \mathrm{mL}) \\
\text { Pre-supplement: } 11.5 \pm 5.8 \\
\text { Post-supplement: } 14.4 \pm 5.2 \\
\text { MCP-1 (pg/mL)Pre-supplement: } \\
126 \pm 36 \\
\text { Post-supplement: } 97.3 \pm 17.9 \\
\text { TBARS (nM) Pre-supplement: } 2.35 \pm 1.11 \\
\text { Post-supplement: } 1.84 \pm 0.89\end{array}$ & $\begin{array}{l}\text { Tomato juice supplementation } \\
\text { resulted in a decrease in levels of the } \\
\text { inflammatory adipokine MCP-1, and } \\
\text { an increase in levels of the } \\
\text { anti-inflammatory adipokine } \\
\text { adiponectin. }\end{array}$ \\
\hline Biddle et al. [61] & $\begin{array}{c}\text { Patients NYHA class II or } \\
\text { III }(n=40)\end{array}$ & $\begin{array}{l}\text { V8 juice containing } 29.4 \mathrm{mg} \text { of } \\
\text { lycopene/day for } 30 \text { days }\end{array}$ & $\begin{array}{c}\text { Control }(n=18) \\
\text { Intervention }(n=22)\end{array}$ & $\begin{array}{c}\text { Plasma Lycopene }(\mu \mathrm{mol} / \mathrm{L}) \\
\text { Control, pre-supl: } 0.56 \\
\text { Control, post-supl: } 0.58 \\
\text { Intervention, pre-supl: } 0.51 \\
\text { Intervention, post-supl: } 0.76\end{array}$ & $\begin{array}{c}\text { Serum CRP }(\mathrm{mg} / \mathrm{L}) \\
\text { Control, pre-supl: } 4.8 \pm 3.4 \text { Control, } \\
\text { post-supl: } 4.5 \pm 3.8 \\
\text { Intervention, pre-supl: } 3.4 \pm 3.1 \\
\text { Intervention, post-supl: } 3.1 \pm 2.8\end{array}$ & $\begin{array}{l}\text { C-reactive protein levels decreased } \\
\text { significantly in the intervention } \\
\text { group in women and but not in men } \\
(p=0.04) .\end{array}$ \\
\hline McEneny et al. [62] & $\begin{array}{l}\text { Moderately overweight, } \\
\text { middle-aged individuals } \\
\qquad(n=54)\end{array}$ & $\begin{array}{l}\text { Control diet ( }<10 \mathrm{mg} \\
\text { lycopene/week)lycopene-rich } \\
\text { diet }(224-350 \mathrm{mg} / \text { week }) \\
\text { lycopene supplement }(70 \\
\text { mg/week)for } 12 \text { weeks }\end{array}$ & $\begin{array}{l}\text { Control } \operatorname{diet}(n=18) \\
\text { Lycopene } \operatorname{diet}(n=18) \\
\text { Lycopene supl }(n=18)\end{array}$ & $\begin{array}{c}\text { Serum Lycopene (mmol/L) } \\
\text { Baseline } \\
\text { Control: } 0.26(0.03) \text { Lycopene } \\
\text { diett } 0.41(0.04) \\
\text { Lycopene supl: } 0.29(0.03) \\
\text { Week } 12 \\
\text { Control: } 0.27(0.03) \text { Lycopene } \\
\text { diet: } 1.14(0.05) \\
\text { Lycopene supl: } 0.87(0.06)\end{array}$ & 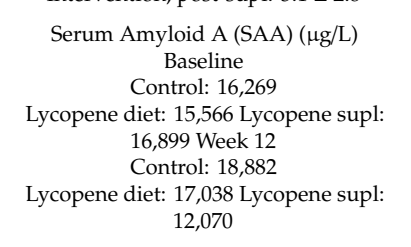 & $\begin{array}{l}\text { Lycopene supplement tended to } \\
\text { produce a greater response in } \\
\text { reducing SAA concentrations and in } \\
\text { influencing HDL's function } \\
\text { compared to the high-tomato diet. }\end{array}$ \\
\hline Petyaev et al. [63] & $\begin{array}{l}\text { Patients with coronary } \\
\text { vascular disease }(n=142)\end{array}$ & $\begin{array}{c}7 \mathrm{mg} \text { of lycopene/day for } 1 \\
\text { month, two different lycopene } \\
\text { supplements }\end{array}$ & $\begin{array}{l}\text { Lactolycopene (L1) }(n=68) \\
\text { Lycosome GA (L2) }(n=74)\end{array}$ & $\begin{array}{l}\text { Serum Lycopene (ng/mg } \\
\text { cholesterol) } \\
\text { Baseline } \\
\text { L1: } 58.0 \mathrm{~L} 2: 55.0 \\
\text { Week } 4 \\
\text { L1: } 87.0 \\
\text { L2: } 237.0\end{array}$ & $\begin{array}{c}\text { CRP }(\mathrm{mg} / \mathrm{L}) / \mathrm{MDA}(\mu \mathrm{M}) \\
\text { Baseline } \\
\text { L1: } 6.0 / 141.0 \\
\text { L2: } 6.8 / 154.0 \\
\text { Week } 4 \\
\text { L1: } 6.2 / 156.0 \\
\text { L2: } 61 / 151.0 \\
\text { hsCRP }(\mathrm{mg} / \mathrm{L}) / \mathrm{IL}-6(\mathrm{pg} / \mathrm{mL}) / \mathrm{TNF}-\mathrm{a}\end{array}$ & $\begin{array}{l}\text { Lycopene supplementation had no } \\
\text { impact on serum CRP level. } \\
\text { Lactolycopene did not affect } \\
\text { inflammatory markers by the end of } \\
\text { the interventional period, whereas } \\
\text { lycosome-formulated lycopene } \\
\text { significantly reduced MDA }\end{array}$ \\
\hline Gajendragadkar et al. [64] & $\begin{array}{l}\text { Statin treated CVD } \\
\text { patients and healthy } \\
\text { controls }(n=72)\end{array}$ & $\begin{array}{l}7 \text { mg lycopene (1) or placebo } \\
\text { (2)/day for } 2 \text { months } \\
\text { Patients }(p) \text { and Healthy (H) }\end{array}$ & $\begin{array}{l}\text { P1: }(n=24) \\
\text { P2: }(n=12) \\
\text { H1: }(n=24) \\
\text { H2: }(n=12)\end{array}$ & $\begin{array}{c}\text { Serum Lycopene }(\mu \mathrm{g} / \mathrm{L}) \\
\text { Baseline/Day } 56 \\
\text { P1: } 146 / 275 \\
\text { P2: } 128 / 178 \\
\text { H1: } 170 / 267 \\
\text { H2: } 182 / 160\end{array}$ & $\begin{array}{c}\text { (pg/mL) Baseline } \\
\text { P1: } 2.13 / 1.54 / 2.13 \\
\text { P2: } 1.15 / 1.120 / 5.55 \\
\text { H1: } 1.15 / 1.32 / 5.39 \\
\text { H2: } 2.83 / 0.92 / 5.55 \\
\text { Day } 56 \\
\text { P1: } 2.37 / 1.51 / 2.37 \\
\text { P2: } 1.186 / 0.92 / 5.65 \\
\text { H1: } 1.87 / 1.02 / 4.92 \\
\text { H2: } 1.65 / 0.84 / 5.32\end{array}$ & $\begin{array}{l}\text { hsCRP, IL-6 and TNF-a levels were } \\
\text { unchanged for lycopene vs. placebo } \\
\text { treatment groups in the CVD arm as } \\
\text { well as the HV arm }\end{array}$ \\
\hline
\end{tabular}


Table 2. Cont

\begin{tabular}{|c|c|c|c|c|c|c|}
\hline Study (Ref) & Study Population & Intervention & Final $n$ & Lycopene Measurement & Inflammation Biomarkers & Conclusions \\
\hline Kim et al. [65] & Healthy men $(n=126)$ & $\begin{array}{c}\text { Placebo }(p) \\
\text { Low lycopene, } 6 \mathrm{mg} / \mathrm{d}(\mathrm{L}) \\
\text { High lycopene, } 15 \mathrm{mg} / \mathrm{d} \text { (H) } \\
\text { For } 8 \text { weeks }\end{array}$ & $\begin{array}{l}p:(n=38) \\
\mathrm{L}:(n=41) \\
\mathrm{H}:(n=37)\end{array}$ & $\begin{array}{c}\text { Serum Lycopene }(\mu \mathrm{g} / \mathrm{mL}) \\
\text { Baseline/8 weeksp: } 0.2 / 0.2 \\
\text { L: } 0.2 / / 2.26 \\
\text { H: } 0.2 / 0.33\end{array}$ & $\begin{array}{c}\text { hsCRP (mg/dL)Baseline/8 weeks } \\
p: 1.14 \pm 0.22 / 1.10 \pm 0.27 \\
\text { L: } 1.39 \pm 0.33 / 1.40 \pm 0.37 \\
\text { H: } 1.25 \pm 0.44 / 0.54 \pm 0.10\end{array}$ & $\begin{array}{l}\text { A reduction in hs-CRP in the } 15-\mathrm{mg} g \\
\text { lycopene/day group and the inverse } \\
\text { correlation between changes in } \\
\text { lycopene and changes in hs-CRP in } \\
\text { this study, suggest that lycopene } \\
\text { may play a role in inflammatory } \\
\text { processes by interfering the action of } \\
\text { cytokines. }\end{array}$ \\
\hline Markovits et al. [66] & $\begin{array}{l}\text { Obese patients }(p) \text { and } \\
\text { healthy controls (C) } \\
\qquad(n=16)\end{array}$ & $\begin{array}{l}\text { Patients received Lyc-o-mato, } \\
30 \mathrm{mg} / \mathrm{d} \text { for } 4 \text { weeks }\end{array}$ & $\begin{array}{l}p:(n=8) \\
C:(n=8)\end{array}$ & $\begin{array}{c}\text { Serum Lycopene }(\mu \mathrm{g} / \mathrm{mL}) \\
\text { C: } 0.14 \pm 0.07 \\
\text { p;baseline: } 0.23 \pm 0.22 \\
p \text {;supple: } 1.15 \pm 0.21\end{array}$ & $\begin{array}{c}\text { CRP }(\mathrm{mg} / \mathrm{L}) / \mathrm{IL}-6(\mathrm{pg} / \mathrm{mL}) / \mathrm{TNF}-\mathrm{a}(\mathrm{pg} / \mathrm{mL}) \\
\text { Baseline } \\
\text { C: } 1.1 / 1.0 / 1.4 p: 6.5 / 3.6 / 1.4 \text { Week } 4 \\
p ; \text { placebo: } 5.5 / 3.5 / 1.4 \\
\text { p; supple: } 5.6 / 4.7 / 1.5\end{array}$ & 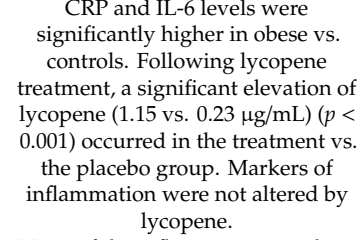 \\
\hline Thies et al. [67] & $\begin{array}{l}\text { Moderately overweight, } \\
\text { disease-free, middle-aged } \\
\text { adults }(n=225)\end{array}$ & $\begin{array}{l}\text { Control diet }(\mathrm{C}) \\
\text { High-tomato diet }(\mathrm{H}) \\
\text { Lycopene capsules }(10 \mathrm{mg} / \mathrm{d}) \\
\text { (L) for } 12 \text { weeks }\end{array}$ & $\begin{array}{l}\text { C: }(n=76) \\
\text { H: }(n=81) \\
\text { L: }(n=68)\end{array}$ & $\begin{array}{c}\text { Plasma Lycopene }(\mu \mathrm{g} / \mathrm{mL}) \\
\text { Baseline/12 weeks C: } 0.4 / 0.4 \\
\text { H: } 0.4 / 1.1 \\
\text { L: } 0.4 / 0.85\end{array}$ & $\begin{array}{c}\text { hsCRP }(\mathrm{mg} / \mathrm{L}) \\
\text { Baseline/12 weeks } \\
\text { C: } 3.18 / 2.08 \\
\text { H: } 1.51 / 1.37 \\
\text { L: } 2.27 / 2.16 \\
\text { IL-6 }(\mathrm{pg} / \mathrm{L}) \\
\text { Baseline/12 weeks C: } 1.37 / 1.38 \\
\text { H: } 1.21 / 1.15 \\
\text { L: } 1.44 / 1.31\end{array}$ & $\begin{array}{l}\text { None of the inflammatory markers } \\
\text { changed significantly after the } \\
\text { dietary intervention. These data } \\
\text { indicate that a relatively high daily } \\
\text { consumption of tomato-based } \\
\text { products (equivalent to } 32-50 \mathrm{mg} \\
\text { lycopene/d) or lycopene } \\
\text { supplements }(10 \mathrm{mg} / \mathrm{d}) \text { is ineffective } \\
\text { at reducing conventional CVD risk } \\
\text { markers in moderately overweight, } \\
\text { healthy, middle-aged individuals. }\end{array}$ \\
\hline Upritchard et al. [68] & $\begin{array}{c}\text { Patients with } \\
\text { well-controlled type } 2 \\
\text { diabetes aged }<75 \text { years } \\
(n=57)\end{array}$ & $\begin{array}{c}\text { Placebo }(\mathrm{C}) \\
\text { Tomato juice } 500 \mathrm{~mL} / \mathrm{d}(\mathrm{T}) \\
\text { for } 4 \text { weeks }\end{array}$ & $\begin{array}{l}\text { C: }(n=13) \\
\text { T: }(n=15)\end{array}$ & $\begin{array}{c}\text { Plasma Lycopene }(\mu \mathrm{mol} / \mathrm{L}) \\
\text { Baseline } / 4 \text { weeks } \\
\text { C: } 0.31 / 0.28 \\
\text { T: } 0.39 / 1.08\end{array}$ & $\begin{array}{c}\text { Plasma CRP }(\mathrm{mg} / \mathrm{L}) \\
\text { Baseline/4 weeks } \\
\text { C: } 3.1 / 3.1 \\
\text { T: } 3.8 / 4.1 \\
\text { L/LC } \\
\text { CRP }(\mathrm{ug} / \mathrm{L}) \\
\text { T-2: } 336.2 / 349.5 \\
\text { T0: } 315.6 / 319.2\end{array}$ & $\begin{array}{l}\text { Plasma lycopene levels increased } \\
\text { nearly three-fold }(p=0.001) \text { and no } \\
\text { significant decreases in plasma levels } \\
\text { of CRP }\end{array}$ \\
\hline Jacob et al. [69] & Healthy subjects $(n=24)$ & $\begin{array}{c}2 \text { weeks depletion } \\
\text { followed by } 2 \text { weeks } \\
\text { tomato juice } 500 \mathrm{~mL} / \mathrm{d}(41 \mathrm{mg} / \mathrm{L} \\
\text { lycopene, } 90 \mathrm{mg} / \mathrm{L} \text { Vitamin C) } \\
\text { (L) or enriched with Vitamin C } \\
(870 \mathrm{mg} / \mathrm{L})(\mathrm{LC})\end{array}$ & $\begin{array}{l}\text { T-2: baseline } \\
\text { T0: after depl. } \\
\text { T + 2: after inter. } \\
\text { L: }(n=12) \\
\text { LC: }(n=12)\end{array}$ & $\begin{array}{c}\text { Plasma Lycopene }(\mu \mathrm{mol} / \mathrm{L}) \\
\text { L/LC } \\
\text { T-2: } 0.72 / 0.71 \\
\text { T0: } 0.42 / 0.34 \\
\text { T + } 2: 1.05 / 0.91\end{array}$ & $\begin{array}{c}\text { T + 2: 262.3/247.1 } \\
\text { IL-1 B (ng/L) T-2: } 3.45 / 12.59 \\
\text { T0: } 3.87 / 10.68 \\
\text { T + 2: } 4.39 / 6.40 \\
\text { TNF-a (ng/L) T-2: } 6.97 / 2.93 \\
\text { TO: } 6.01 / 3.35 \\
\text { T + 2: } 3.45 / 3.28 \\
\text { MDA ( } \mu \mathrm{mol} / \mathrm{L}) \mathrm{T}-2: 0.55 / 0.60 \\
\text { To: } 0.54 / 0.56 \\
\text { T + 2: } 0.53 / 0.50\end{array}$ & $\begin{array}{c}\text { The consumption of tomato juice led } \\
\text { to a reduction of CRP in both groups } \\
\text { All other markers were affected to a } \\
\text { lesser extent or remained } \\
\text { unchanged. }\end{array}$ \\
\hline Williams et al. [70] & COPD patients $(n=11)$ & $\begin{array}{c}\text { Rosuvastatin }(20 \mathrm{mg} / \text { day }) \text { for } 4 \\
\text { weeks then a combination of } \\
\text { rosuvastatin ( } 20 \mathrm{mg} / \text { day), DHA } \\
\text { and EPA ( } 1.5 \mathrm{~g} / \text { day }) \text { and } \\
\text { lycopene }(45 \mathrm{mg} / \text { day }) \text { for } \\
8 \text { weeks. }\end{array}$ & $\begin{array}{l}\text { T1: baseline } \\
\text { T2: rosuvastatin } \\
\text { T3: lycopene }\end{array}$ & $\begin{array}{c}\text { Plasma Lycopene }(\mathrm{mg} / \mathrm{L}) \\
\text { T1: } 0.30(0.13-0.54) \text { T2: } 0.56 \\
(0.14-0.77) \\
\text { T3: } 0.50(0.22-0.96)\end{array}$ & $\begin{array}{c}\text { CRP (mg/L) T1: } 3.9(1.9-7.9) \\
\text { T2: } 3.3(0.7-7.6) \\
\text { T3 } 3.8(1.3-8.9) \\
\text { IL-6 (pg/mL) T1: } 2.2(1.6-3.0) \\
\text { T2: } 3.2(2.3-5.1) \\
\text { T3: } 3.1(1.6-4.8)\end{array}$ & $\begin{array}{l}\text { Treatment interventions did not } \\
\text { significicantly change plasma } \\
\text { carotenoid levels. However, there } \\
\text { was a trend for increased lycopene } \\
\text { concentration at visit } 2 \text { and } 3 . \\
\text { Following the interventions, plasma } \\
\text { IL-6 } 6 \text { and CRP were unchanged. }\end{array}$ \\
\hline
\end{tabular}


Table 2. Cont

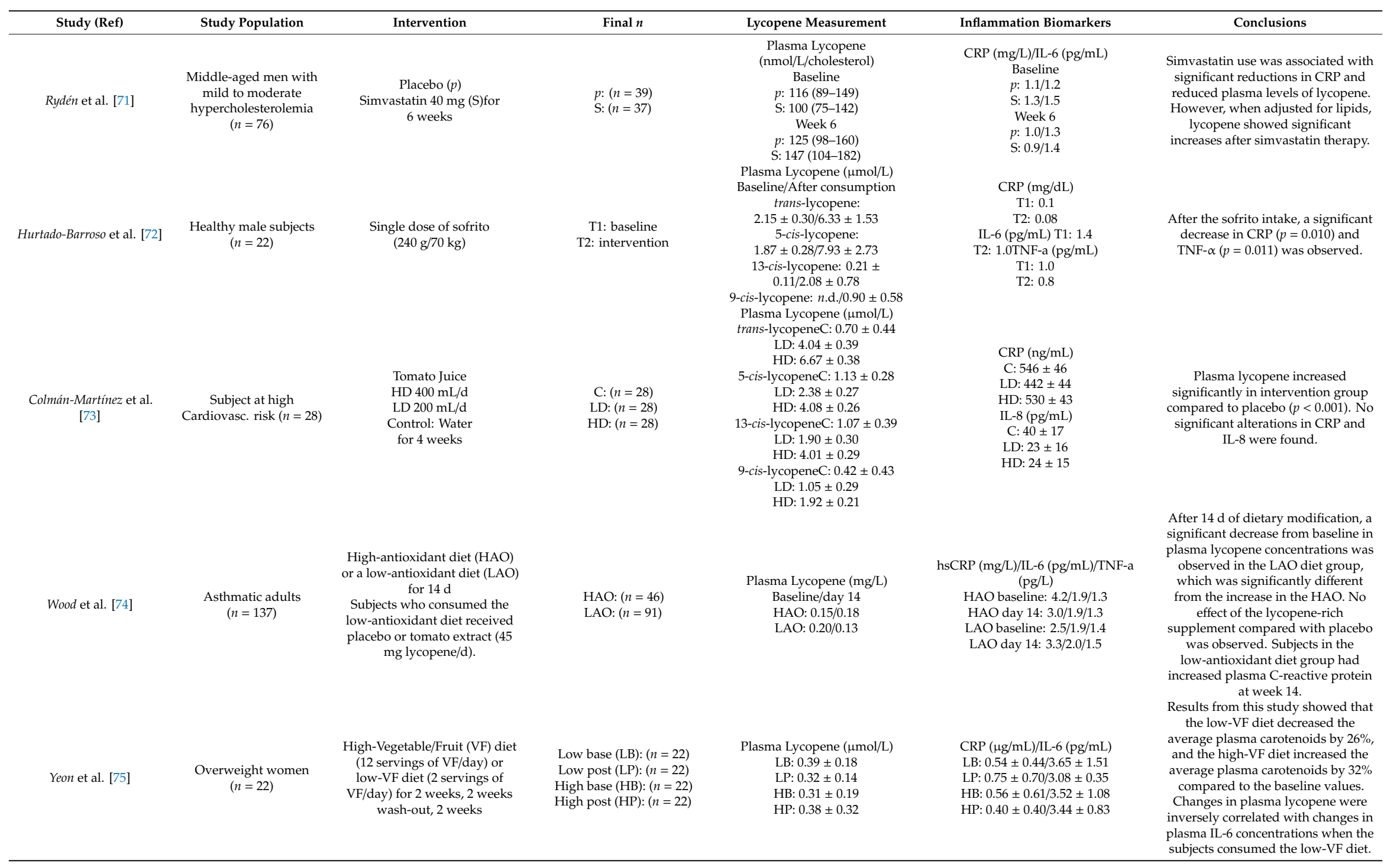




\section{Results}

\subsection{Study Characteristics}

Of the 80 articles identified, screened, and considered for systematic review, 35 articles met the inclusion criteria for critical analysis. All papers were published between 1996 and 2018. Eighteen of the 35 studies used a cross-sectional study design and the remaining 17 were intervention trials. Studies varied widely not only in design and lycopene measurements, but also in the assessment of inflammation biomarkers. While some studies have identified multiple outcome measures, only the measurements of circulating lycopene and inflammatory biomarkers are highlighted in this review. Furthermore, studies that examined possible correlations or made conclusions regarding the relationship between circulating lycopene and inflammatory biomarkers, were also included in this systematic review. In various studies, the reported sample size for the outcomes of interest differed from the total number of participants. As such, these unique sample sizes have been reported with the corresponding measurement (Tables 1 and 2).

\subsection{Cross-Sectional Studies}

As shown in Tables 1 and 2, the discussion of the 35 articles is separated by study design. As displayed in Table 1, the results reported in the 18 included cross-sectional studies reviewed were grouped according to the following categories: type of lycopene measurement, assessment of inflammation biomarkers, and conclusions drawn from the study.

Five of these studies classified participants based on CRP concentrations or lycopenelevels [41,43,45,51,53]. A study by Mazidi et al. [41] divided participants in quartiles depending on CRP concentrations and concluded that a higher lycopene level for each $\mu \mathrm{mol} / 1$ correlated with $0.067 \mathrm{mg} / \mathrm{dl}$ lower CRP levels. Kritchevsky et al. [51] divided participants in tertiles depending on CRP levels and concluded that participants in the higher tertile CRP had significantly lower circulating lycopene levels. Furthermore, Boosalis et al. [53] divided elderly women (77-99 years) into two groups, based on either normal or elevated CRP levels, and showed that the presence of elevated CRP resulted in a significant decrease of lycopene concentrations. In addition, Kim et al. [43] divided healthy women (31-75 years) into tertiles according to serum lycopene concentrations, and reported that subjects in the highest tertile showed significantly lower CRP levels compared to those individuals in the lowest tertile. On the contrary, this association was not found in a study [45] in which young adults were divided into quartiles depending on the lycopene concentrations.

Thirteen other studies assessed the relationship between circulating lycopene and inflammation in healthy participants or patients. These studies report lower circulating lycopene concentrations and higher inflammation biomarker levels in patients with colorectal adenocarcinoma [42,55], carotid artery disease [44], stable angina pectoris [49,57], ischemic stroke [56], chronic hepatitis C [50], gastrointestinal cancer [52], benign prostate hyperplasia, localized and metastatic prostate cancer [54,55] and breast cancer [55] compared to healthy controls [48]. In addition, this relationship was also observed in critically ill patients [58], elderly disabled women [46], and people exposed to Schistosoma [47].

In general, these results suggest that lycopene levels are adversely affected during inflammation and homeostatic imbalance. These cross-sectional data do not clarify the biological relationship between lycopene and inflammation biomarkers. However, they do indicate the extent to which lycopene is associated with inflammation. They also indicate that the depletion of lycopene may be, in part, the first signs of low-grade inflammation.

\subsection{Intervention Studies}

As displayed in Table 2, the results reported in the 17 included intervention studies reviewed were grouped according to the following categories: type of lycopene measurement, assessment of inflammation biomarkers, type of intervention and conclusions drawn from the study. 
Each of the included 17 intervention studies assessed lycopene levels and inflammatory biomarkers pre- and postintervention. All studies, except one, reported increased circulating lycopene levels following tomato/lycopene supplementation. In the exceptional study, supplementation with Lactolycopene capsules (supplements with lycopene entrapped with whey proteins) did not lead to a significant increase, but supplementation with Lycosome GA capsules (supplements with microencapsulated lycopene) did [63]. In a second study, supplementation with a combination of lycopene and rosuvastatin also did not significantly change plasma lycopene levels [70].

In ten intervention studies, biomarkers of inflammation were not reported to change after tomato/lycopene supplementation [59,62,64,66-68,70,73-75]. On the contrary, in a study by Li et al. [60], tomato juice supplementation led to a decrease of inflammatory adipokine MCP-1, and an increase in anti-inflammatory adiponectin levels in healthy Taiwanese females (20-30 years). Additionally, Biddle et al. [61] reported that tomato juice supplementation significantly decreased CRP levels in female heart failure patients, but not in male patients. Conversely, a decrease in hs-CRP was observed in healthy men following high lycopene (15 mg/day) supplementation [65] and after a single dose of tomato sauce (sofrito) [72].

Petyaev et al. [63] investigated the effect of supplementation with Lactolycopene or Lycosome GA capsules in patients with coronary artery disease. Serum lycopene levels of participants receiving Lactolycopene did not increase and CRP and MDA levels did not change after one month of supplementation. Nevertheless, in the group that received Lycosome GA capsules, circulating lycopene increased after one month, but only MDA was significantly reduced. In addition, opposite results were observed in healthy subjects in a study by Jacob et al. [69] in which CRP levels decreased following tomato juice supplementation, but IL-1 $\beta, \mathrm{TNF}-\alpha$, and MDA levels remained stable.

Four of the seventeen selected studies conducted intervention studies in moderately overweight or obese individuals. Biomarkers for inflammation are often elevated in obese individuals compared to healthy individuals. One study [66] also used a healthy control group and concluded that pre-intervention CRP and IL-6 levels were significantly higher in obesity versus controls. All four concluded that markers of inflammation were not altered by lycopene, despite the significant increase in circulating lycopene after supplementation $[62,66,67,75]$.

Four of the selected intervention studies investigated the effect of lycopene supplementation on inflammatory markers in patients with Cardiovasc. diseases [61,63,64,73]. These studies did not show consistent results. In one study, only MDA decreased [63]. In the next study, only CRP decreased in women (not in men) [61]. In the other two studies no alterations in inflammation biomarkers were observed after supplementation with lycopene [64,73]. The latter results were also observed in a study conducted in patients with type 2 diabetes, in which plasma lycopene levels increased nearly three-fold $(p=0.001)$, but no significant decreases in plasma levels of CRP were observed [68].

Six included intervention studies evaluated possible associations between lycopene/tomato supplementation and inflammation in healthy participants. In three of these studies, markers of inflammation did not change after supplementation, although circulating lycopene had increased by about 50 percent $[59,64,69]$. However, in another study the lycopene concentration also increased 1.5 times, and a significant decrease in hs-CRP was observed [65]. Furthermore, Hurtado-Barroso et al. [72] observed a three-fold increase in circulating lycopene and a significant decrease in CRP after a single dose of tomato sauce (sofrito). It is worth mentioning that CRP values in both studies were already below standard values before the start of the intervention. Li et al. [60] demonstrated that tomato juice supplementation led to a decrease of inflammatory adipokine MCP-1, and an increase in anti-inflammatory adiponectin levels in healthy young Taiwanese females. Compared to the other studies in which no or minor effects were seen on CRP, MCP-1 and adiponectin may be more sensitive biomarkers and therefore more suitable for studying inflammation in healthy individuals.

Rydén et al. [71] investigated the effect of simvastatin therapy on plasma lycopene levels and inflammatory markers in middle-aged men with mild to moderate hypercholesterolemia. Lycopene levels per total cholesterol (expressed as lycopene/total cholesterol) were significantly increased by 
simvastatin treatment. The findings may indicate that atherogenic lipoprotein particles have improved their antioxidant status through enrichment of carotenoids during simvastatin therapy.

Overall, most studies reported increased circulating lycopene levels after tomato/lycopene supplementation, but less than half of them observed alterations in inflammation biomarkers. In addition, two studies examined the effects of a low antioxidant diet in overweight women and asthmatic adults and observed a decrease in circulating lycopene and an increase in CRP [74,75]. Compared to supplementation, lycopene depletion appears to increase inflammation.

\section{Discussion}

This is, to our knowledge, the first systematic review to assess the correlation and causation between circulating lycopene (the bioavailable lycopene following consumption) and low-grade chronic inflammation. This review reveals that there is strong evidence indicating that lower circulating lycopene concentrations are related with higher inflammation biomarkers in patients with various diseases. In addition, this systematic review shows that there is little evidence that tomato intake or lycopene supplementation diminishes this inflammation.

In only one of the five studies in which CRP or lycopene levels were arranged into tertiles/quartiles, no association was found between circulating lycopene and CRP $[41,43,45,51,53]$. This could be attributable to the low CRP levels of the studied young adults (18-30); all mean CRP levels measured were between 0.99 and $1.11 \mathrm{mg} / \mathrm{L} \mathrm{[45].} \mathrm{On} \mathrm{the} \mathrm{contrary,} \mathrm{the} \mathrm{results} \mathrm{from} \mathrm{another} \mathrm{study} \mathrm{[43]}$ showed a significant association and measured high-sensitivity CRP (hs-CRP) ranging from 0.80 and $1.27 \mathrm{mg} / \mathrm{L}$. Moreover, when comparing the corresponding lycopene levels, it is striking that the values of Hozawa et al. [45] lie between 0.0242 and $0.0918 \mu \mathrm{mol} / \mathrm{L}$, whereas most lycopene levels measured in all studies are between 0.1 and $1 \mu \mathrm{mol} / \mathrm{L}$. It is therefore also possible that a non-reliable lycopene measurement has been carried out, so that no association could be found. The other three studies [41,51,53] did confirm the findings of Kim et al. [43], so there is strong evidence to suggest an association between circulating lycopene and CRP.

The eighteen studies evaluating the relationship between circulating lycopene and inflammation in healthy participants and patients gave similar results. These studies found lower circulating lycopene concentrations coincide with higher inflammation biomarkers in patients suffering from various diseases. These comparable results suggest that lycopene levels are adversely affected during inflammation and disturbed homeostasis. One possible explanation is that the development of oxidative stress during inflammation is responsible for the decreased lycopene levels. The prooxidant-antioxidant imbalance that ensues during oxidative stress may result in the increased utilization of endogenous and exogenous antioxidants, depleting circulating antioxidant concentrations. For that reason, any protective association that exists between serum lycopene and inflammation in patients may be attenuated [76-79]. Although the mechanisms underpinning reduced lycopene levels during inflammation are not fully elucidated, depletion of lycopene may be in part the first sign of low-grade inflammation.

Seventeen intervention studies were identified which better elucidate this carotenoid's causal effect on inflammation and outcomes. Results from cross-sectional studies preclude the ability to ascribe causality because of both potential confounding and a lack of knowledge about the temporal relation between variables of interest. Most studies successfully increased lycopene levels through supplementation or tomato intake. In one study, supplementation with Lactolycopene capsules did not significantly increase lycopene levels. The authors emphasized the importance of proper supplement development, as another supplement increased circulating lycopene. In addition, supplementation with a combination of lycopene and rosuvastatin did not increase lycopene levels either [70]. The latter result could be explained by another study, in which supplementation with simvastatin, a comparable statin, led to a decrease in circulating lycopene. However, lycopene levels per total cholesterol were significantly increased following simvastatin treatment. The observed change in carotenoid status during simvastatin treatment was mainly attributed to the decrease in cholesterol, emphasizing the importance of cholesterol adjustment for expressing carotenoid levels [71]. 
This review found that the effect of lycopene supplementation or tomato intake on inflammation is incongruent: no changes in inflammation biomarkers were observed in half of the studies, and in the other half not all results were in line. Inflammatory markers were not altered by lycopene in moderately overweight or obese people, despite the significant increase in circulating lycopene after supplementation [62,66,67]. Intervention studies in patients with Cardiovascular disease or type 2 diabetes also showed minimal reduction of inflammatory markers $[61,63,64,68]$. In some intervention studies, it was stated that the intervention period was too short to observe a decrease in inflammatory biomarkers in patients. However, previous research has shown that treatment with non-steroidal anti-inflammatory drugs (NSAIDs) for a short period (two weeks) may reduce inflammatory biomarkers in patients, so these inflammatory biomarkers are unlikely to take longer to decrease $[80,81]$. Likewise, the results of lycopene supplementation in healthy participants were also inconsistent. Only two studies observed a significant decrease in hs-CRP after high lycopene supplementation (15 mg/day) or tomato sauce (sofrito) intake [72], but no effects were found after low lycopene supplementation (6 mg/day) [65] nor $7 \mathrm{mg} /$ day [64]. The hs-CRP test accurately measures low CRP levels to identify low but persistent inflammatory levels. Therefore, it is more suitable for studying low-grade chronic inflammation in healthy participants in further research. However, it is debatable whether such a significant reduction in CRP below the standard values of $1-3 \mathrm{mg} / \mathrm{L}$ is clinically relevant and shows an actual anti-inflammatory effect, as these low CRP values already demonstrate that there is hardly any inflammation present. The other studies evaluating CRP report no significant changes in CRP levels following lycopene intake, probably because of the already low basal value in healthy participants. In addition, it would be of interest to evaluate new, more sensitive biomarkers in subsequent studies, as MCP-1 and adiponectin prove to be suitable biomarkers to study inflammation in healthy subjects [60].

Two intervention studies investigated the potential beneficial effects of lycopene in its isolated form (supplement) and via a lycopene-rich diet. These particular studies showed that both methods were successful in increasing circulating lycopene, but not in changing inflammation biomarkers [62,67]. These results suggest that the form in which lycopene is administered is of less importance than the absorption per se. For example, the absorption of lycopene can be improved by method of preparation such as adding olive oil [82]. Current literature indicates that the incorporation of a functional food with the compound of interest could potentially enhance these protective properties through the provision of an intact food matrix. However, more research is needed to elucidate these speculations. The matrix may provide a synergistic environment to promote the bioactivity of phytonutrients. However, this matrix also presents a challenge, since the direct effects of lycopene cannot be separated from other bioactive compounds within the food $[83,84]$.

\subsection{Molecular Mechanisms of Action}

The incongruent results observed between the cross-sectional and intervention studies may be attributed to the different mechanisms of action of lycopene. Many in vitro studies elucidated the protective properties of carotenoids. As free radical scavengers, carotenoids react with reactive oxygen species (ROS) by three distinct mechanisms: (i) radical addition/adduct formation, (ii) electron transfer, and (iii) allylic hydrogen abstraction [85]. However, it is difficult to extrapolate the results of such studies because processes in the human body are more complex. It is probable that a number of factors may serve to decrease the antioxidant effectiveness of carotenoids in vivo, making them ineffective against certain ROS [86]. Furthermore, recent findings have shown that the participation of phytochemicals in redox metabolism is far more complicated than simply scavenging free radicals and avoiding oxidation of molecules. The cellular redox homeostasis is sustained by an overall and well-adjusted network of subcellular redox circuits that oscillate constantly depending on nutrients and energy supplies, genetic and epigenetic codes, and interactions with the external environment [87].

Barros et al. [87] suggest the hypothetical existence of the $1 \mathrm{NAD}(p)+/ \mathrm{NAD}(p) \mathrm{H}$-responsive redox switch of eukaryotic cells that triggers distinct phenotypic fates depending upon cellular 
redox balance. This theory may explain the reduced lycopene levels in impaired situations as well as the paradoxical phenomenon where depletion of lycopene appears to increase inflammation, but lycopene supplementation does not decrease inflammation.

This theory suggests that an increase of the cellular antioxidant capacity (from dietary intake or generated endogenously) slides the antioxidant "seesaw" pivot point to the right, attenuating the magnitude of ROS/RNS production in the cell. However, an excessive antioxidant load in cells (sliding further to the right) could prevent beneficial processes mediated by the Nrf2-Keap1-EpRE system (Figure 2) [87,88]. Halliwell [89] describes the "antioxidant paradox" that supports this theory. The term "antioxidant paradox" is often used to refer to the observation that oxygen radicals and other ROS are implicated in several human diseases, but giving large doses of dietary antioxidants to human subjects has, in most studies, little or no preventative or therapeutic effect on inflammation. In addition, providing weak pro-oxidants to manipulate endogenous antioxidant levels may be a more useful approach for prevention of non-communicable diseases than is consumption of large doses of dietary antioxidants [89]. For example, it is well-known that physical activity increases the level of oxidative stress, but this appears to be beneficial to health. This same stimulus is in fact necessary to allow upregulation of endogenous antioxidant defenses, a phenomenon known as hormesis [90-92]. In addition to physical activity, various phytochemicals present in fruits and vegetables also can increase the level of oxidative stress and may exert health effects in other ways than lycopene.

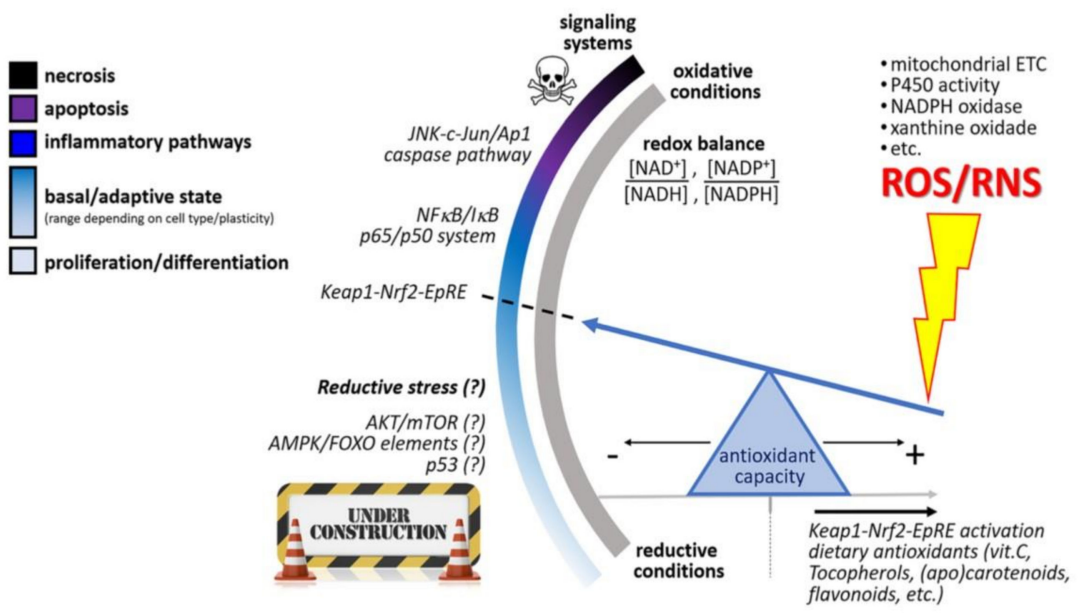

Figure 2. Hypothetical $\mathrm{NAD}(p)+/ \mathrm{NAD}(p) \mathrm{H}$-responsive redox switch of eukaryotic cells that triggers distinct phenotypic fates depending upon cellular redox balance. From a basal condition (optimum redox balance), the redox switch elicits inflammatory pathways, apoptosis, or necrosis, following increasing oxidative conditions, whereas unclear "reductive stress" mechanisms are triggered when $\mathrm{NAD}(p) \mathrm{H}$ coenzymes prevail in cellular compartments. An increase of the cellular antioxidant capacity (from diet intake or generated endogenously) slides the antioxidant "seesaw" pivot point to the right, attenuating the magnitude of ROS/RNS production in the cell. However, an excessive antioxidant load in cells (sliding further to the right) could prevent beneficial processes mediated by the Nrf2-Keap1-EpRE system. This figure was adapted from reference [87].

Isothiocyanates from cruciferous vegetables can react directly with sulfhydryl residues of Keap1, causing the release of Nrf2. The ROS scavenging capacity of curcumin from turmeric is mainly attributed to its structure as a bis- $\alpha, \beta$-unsaturated $\beta$-diketone of the two ferulic acid units, connected through a methylene group, which in addition can modify the thiol groups of Keap1, causing the release of Nrf2 [93]. The semi-synthetic flavonoid 7-mono-O-( $\beta$-hydroxyethyl)-rutoside (monoHER) acts as a double-edged sword in cells subjected to oxidative stress; the antioxidant offers direct protection by scavenging ROS and the oxidized monoHER adducts Keap1, causing the release of Nrf2 [94]. Alternatively, epigallocatechin gallate (from tea), cinnamaldehyde (from cinnamon), and resveratrol (from grapes) act on upstream kinases such as Akt, ERK, PI3K, PKC, and JNK 
causing the indirect release of Nrf2 from Keap1 [93]. Nrf2 is translocated to the nucleus and binds to the antioxidant response element (ARE) located in the promoters of genes coding for antioxidant and detoxifying enzymes. Nrf2/ARE-dependent genes code for several mediators of the antioxidant response, including glutathione S-transferases (GSTs), thioredoxin, $\mathrm{NAD}(p) \mathrm{H}$ quinone oxidoreductase 1 (NQO-1), and heme oxygenase 1 (HO-1) [95]. Paradoxically, this reaction is considered weakly pro-oxidant [96]. The resulting oxidative stress supports the hormetic feedback and therefore leads to an endogenous increase in antioxidant defenses. It is possible that an excess of exogenous antioxidants may have detrimental effects on health by blocking the hormetic process $[90,91]$. In addition, various phytochemicals, such as the flavonoid quercetin from onions, can increase the endogenous antioxidant defenses in multiple ways. Similar to monoHER, oxidation products of quercetin are able to modify the thiol groups of Keap1, causing the release of Nrf2 and by up-regulation of Nrf2 through the regulation of both transcription and posttranscription sites and repression of Keap1 by affecting the posttranscription site $[97,98]$.

\subsection{Dietary Recommendations}

A wealth of epidemiological evidence indicates that diets rich in plant products (grains, fruits and vegetables) contribute to overall health $[2-4,99,100]$. It is not clear whether this health-promoting effect is mainly attributable to the antioxidants in these plant products. However, the evidence suggests that antioxidants do play an important role in maintaining health: two studies included in this review examined a low antioxidant diet, during which a decrease in circulating lycopene and a subsequent increase in CRP was observed [74,75]. The available data thereby imply that it is beneficial to consume lycopene-rich foods occasionally to stay healthy and keep circulating lycopene at a basal level. It is preferable to consume lycopene through whole food sources such as tomatoes, rather than to ingest it through supplementation. This is because (i) lycopene is stable during preparation methods, (ii) other phytonutrients are also present in e.g.; tomatoes, (iii) the potential benefits of the food matrix, and (iv) the costs. But as this study shows, it is unlikely that taking additional lycopene will help restore health if inflammation is already present. Nevertheless, additional research is needed to determine evidence-based recommendations on the effect of long-term lycopene intake or supplementation and reduction of inflammation. In today's society, antioxidants are considered healthy, partly because of results from in vitro studies. It is possible that the health effects of fruit and vegetables are due to the wide variety of bioactive substances in the food matrices and the synergy between the different mechanisms of action of these phytochemicals in the body. Nevertheless, the riddle of the "antioxidant paradox," as described in Section 4.1, is yet to be fully deciphered. Phytochemicals in fruits and vegetables, both anti- and pro-inflammatory, appear to play a key role in this. In further research, it is important to consider the complexity of the endogenous antioxidant defense system [90]. Epidemiological evidence indicates that a multifactorial strategy of exercise, a healthy weight, no smoking, and a balanced diet that includes plenty of fruits, grains, and vegetables, is optimal to prevent low-grade chronic inflammation and maintain health overall [89].

\subsection{Strengths and Limitations}

This systematic review is one of the first studies that focusses on studying circulating lycopene measurements and its effect on inflammation, instead of merely the intake of lycopene. Self-reported measures of lycopene intake are subject to recall bias or memory errors, and do not provide insights into the body's absorption, distribution, metabolism, and excretion of lycopene. Another strength of this study is that in this review, a clear distinction is made between effects reported in observational studies versus intervention studies. These reported effects are furthermore explained by incorporating details on the molecular mechanism of action of lycopene. The results of the cross-sectional studies are consistent with the findings of previous systematic reviews assessing the relationship between lycopene and vascular risk, metabolic syndrome, prostate, and bladder cancer [33-36]. 
However, suggestions made in these reviews about the effect of lycopene supplementation to reduce the risk of these diseases differ from the findings in this review, as this review highlights that there is little evidence that lycopene supplementation reduces inflammation.

Furthermore, this study is not without limitations. Overall, intervention studies were characterized by small sample sizes and short duration. In follow-up research, it would be of interest to investigate the effects of long-term lycopene supplementation on inflammation. Additionally, it is important to acknowledge that there might be publication bias in the intervention studies. It is known that positive results are published in scientific literature more often than negative or inconclusive ones. This study has also not been able to perform a meta-analysis to quantify the potential effects of lycopene because studies differed widely in lycopene and inflammatory biomarkers measurements. Lastly, even though all authors were involved in conducting the systematic search, setting inclusion criteria, and reviewing the inclusion of publications, selection bias may have affected the in- and exclusion of certain studies.

\section{Conclusions}

The available evidence indicates that lycopene levels are adversely affected during inflammation and homeostatic imbalance. Although the mechanisms underpinning these reduced lycopene levels are not fully elucidated, depletion of lycopene may be one of the first signs of low-grade inflammation. Even though supplementation with lycopene or an increased intake of tomatoes does result in an increase in circulating lycopene, there is little evidence that the lycopene increase also results in relieving this inflammation. This phenomenon, also known as the "antioxidant paradox," limits the added value of lycopene supplementation in both patients and healthy individuals.

Author Contributions: H.P.v.S.: Conceptualization, formal analysis, investigation, writing-original draft; A.B.: Supervision, conceptualization, validation, writing-review \& editing; A.d.B.: Funding acquisition, supervision, conceptualization, formal analysis, validation, writing-review \& editing. All authors have read and agreed to the published version of the manuscript.

Funding: This research was funded by the Dutch Topsector Tuinbouw \& Uitgangsmaterialen, grant number TU1118.

Acknowledgments: The authors thank Tamara Verhoeven and Herman Peppelenbos for their contributions. The authors would like to thank Madhura Rao for constructive criticism of the manuscript. This research has been made possible with the support of the Dutch Province of Limburg.

Conflicts of Interest: The authors declare no conflict of interest.

\section{References}

1. Georgiou, N.; Garssen, J.; Witkamp, R. Pharma-nutrition interface: The gap is narrowing. Eur. J. Pharmacol. 2011, 651, 1-8. [CrossRef] [PubMed]

2. Bagetta, D.; Maruca, A.; Lupia, A.; Mesiti, F.; Catalano, R.; Romeo, I.; Moraca, F.; Ambrosio, F.; Costa, G.; Artese, A.; et al. Mediterranean products as promising source of multi-target agents in the treatment of metabolic syndrome. Eur. J. Med. Chem. 2020, 186, 111903. [CrossRef] [PubMed]

3. Kashi, D.; Shabir, A.; Da Boit, M.; Bailey, S.; Higgins, M. The Efficacy of Administering Fruit-Derived Polyphenols to Improve Health Biomarkers, Exercise Performance and Related Physiological Responses. Nutrients 2019, 11, 2389. [CrossRef]

4. Maria, L.; Eamon, E.; Mana, S.; Rosa, C. Relation of Fruits and Vegetables with Major Cardiometabolic Risk Factors, Markers of Oxidation, and Inflammation. Nutrients 2019, 11, 2381.

5. Bosma-den Boer, M.; van Wetten, M.; Pruimboom, L. Chronic inflammatory diseases are stimulated by current lifestyle: How diet, stress levels and medication prevent our body from recovering. Nutr. Metab. 2012, 9, 32. [CrossRef]

6. de Boer, A.; van de Worp, W.; Hageman, G.; Bast, A. The effect of dietary components on inflammatory lung diseases-a literature review. Int. J. Food Sci. Nutr. 2017, 68, 771-787. [CrossRef] [PubMed]

7. Ruiz-Núñez, B.; Pruimboom, L.; Dijck-Brouwer, D.; Muskiet, F. Lifestyle and nutritional imbalances associated with Western diseases: Causes and consequences of chronic systemic low-grade inflammation in an evolutionary context. J. Nutr. Biochem. 2013, 24, 1183-1201. [CrossRef] [PubMed] 
8. Todoric, J.; Antonucci, L.; Karin, M. Targeting Inflammation in Cancer Prevention and Therapy. Cancer Prev. Res. 2016, 9, 895-905. [CrossRef]

9. Medzhitov, R. Inflammation 2010: New Adventures of an Old Flame. Cell 2010, 140, 771-776. [CrossRef]

10. Calder, P.; Ahluwalia, N.; Albers, R.; Bosco, N.; Bourdet-Sicard, R.; Haller, D.; Holgate, S.; Jönsson, L.; Latulippe, M.; Marcos, A.; et al. A Consideration of Biomarkers to be Used for Evaluation of Inflammation in Human Nutritional Studies. Br. J. Nutr. 2013, 109, S1-S34. [CrossRef]

11. Chen, L.; Deng, H.; Cui, H.; Fang, J.; Zuo, Z.; Deng, J.; Li, Y.; Wang, X.; Zhao, L. Inflammatory responses and inflammation-associated diseases in organs. Oncotarget 2017, 9, 7204-7218. [CrossRef]

12. Del Giudice, M.; Gangestad, S. Rethinking IL-6 and CRP: Why they are more than inflammatory biomarkers, and why it matters. Brain Behav. Immun. 2018, 70, 61-75. [CrossRef] [PubMed]

13. Minihane, A.; Vinoy, S.; Russell, W.; Baka, A.; Roche, H.; Tuohy, K.; Teeling, J.; Blaak, E.; Fenech, M.; Vauzour, D.; et al. Low-grade inflammation, diet composition and health: Current research evidence and its translation. Br. J. Nutr. 2015, 114, 999-1012. [CrossRef] [PubMed]

14. Lacourt, T.; Vichaya, E.; Chiu, G.; Dantzer, R.; Heijnen, C. The High Costs of Low-Grade Inflammation: Persistent Fatigue as a Consequence of Reduced Cellular-Energy Availability and Non-adaptive Energy Expenditure. Front. Behav. Neurosci. 2018, 12, 78. [CrossRef] [PubMed]

15. Liu, C.; Abrams, N.; Carrick, D.; Chander, P.; Dwyer, J.; Hamlet, M.; Macchiarini, F.; PrabhuDas, M.; Shen, G.; Tandon, P.; et al. Biomarkers of chronic inflammation in disease development and prevention: Challenges and opportunities. Nat. Immunol. 2017, 18, 1175-1180. [CrossRef] [PubMed]

16. Mendes, A.; Cruz, M.; Gualillo, O. Editorial: The Physiology of Inflammation-The Final Common Pathway to Disease. Front. Physiol. 2018, 9, 1741. [CrossRef]

17. Kaluza, J.; Håkansson, N.; Harris, H.; Orsini, N.; Michaëlsson, K.; Wolk, A. Influence of anti-inflammatory diet and smoking on mortality and survival in men and women: Two prospective cohort studies. J. Int. Med. 2018, 285, 75-91. [CrossRef]

18. Khoo, H.; Prasad, K.; Kong, K.; Jiang, Y.; Ismail, A. Carotenoids and Their Isomers: Color Pigments in Fruits and Vegetables. Molecules 2011, 16, 1710-1738. [CrossRef]

19. Schieber, A.; Carle, R. Occurrence of carotenoid cis-isomers in food: Technological, analytical, and nutritional implications. Trends Food Sci. Technol. 2005, 16, 416-422. [CrossRef]

20. Rao, A.; Ray, M.; Rao, L. Lycopene. Adv. Food Nutr. Res. 2006, 51, 99-164.

21. Palozza, P.; Parrone, N.; Catalano, A.; Simone, R. Tomato Lycopene and Inflammatory Cascade: Basic Interactions and Clin.Implications. Curr. Med. Chem. 2010, 17, 2547-2563. [CrossRef] [PubMed]

22. Di Mascio, P.; Kaiser, S.; Sies, H. Lycopene as the most efficient biological carotenoid singlet oxygen quencher. Arch. Biochem. Biophys. 1989, 274, 532-538. [CrossRef]

23. Parker, R. Absorption, metabolism, and transport of carotenoids. FASEB J. 1996, 10, 542-551. [CrossRef] [PubMed]

24. Stahl, W.; Sies, H. Lycopene: A Biologically Important Carotenoid for Humans? Arch. Biochem. Biophys. 1996, 336, 1-9. [CrossRef] [PubMed]

25. Gärtner, C.; Stahl, W.; Sies, H. Lycopene is more bioavailable from tomato paste than from fresh tomatoes. Am. J. Clin. Nutr. 1997, 66, 116-122. [CrossRef] [PubMed]

26. van Breemen, R.; Xu, X.; Viana, M.; Chen, L.; Stacewicz-Sapuntzakis, M.; Duncan, C.; Bowen, P.; Sharifi, R. Liquid Chromatography-Mass Spectrometry of cis and all-trans-Lycopene in Human Serum and Prostate Tissue after Dietary Supplementation with Tomato Sauce. J. Agric. Food Chem. 2002, 50, 2214-2219. [CrossRef]

27. Zaripheh, S.; Erdman, J. The Biodistribution of a Single Oral Dose of [14C]-Lycopene in Rats Prefed Either a Control or Lycopene-Enriched Diet. J. Nutr. 2005, 135, 2212-2218. [CrossRef]

28. Wang, X. Lycopene metabolism and its biological significance. Am. J. Clin. Nutr. 2012, 96, S1214-S1222. [CrossRef]

29. Nagao, A. Oxidative Conversion of Carotenoids to Retinoids and Other Products. J. Nutr. 2004, 134, S237-S240. [CrossRef]

30. Huang, C.; Fan, Y.; Lin, C.; Hu, M. Lycopene inhibits matrix metalloproteinase-9 expression and down-regulates the binding activity of nuclear factor-kappa B and stimulatory protein-1. J. Nutr. Biochem. 2007, 18, 449-456. [CrossRef] 
31. Fenni, S.; Hammou, H.; Astier, J.; Bonnet, L.; Karkeni, E.; Couturier, C.; Tourniaire, F.; Landrier, J. Lycopene and tomato powder supplementation similarly inhibit high-fat diet induced obesity, inflammatory response, and associated metabolic disorders. Mol. Nutr. Food Res. 2017, 61, 1601083. [CrossRef] [PubMed]

32. Cha, J.; Kim, W.; Ha, A.; Kim, M.; Chang, M. Anti-inflammatory effect of lycopene in SW480 human colorectal cancer cells. Nutr. Res. Pract. 2017, 11, 90. [CrossRef] [PubMed]

33. Senkus, K.; Tan, L.; Crowe-White, K. Lycopene and Metabolic Syndrome: A Systematic Review of the Literature. Adv. Nutr. 2018, 10, 19-29. [CrossRef] [PubMed]

34. Cheng, H.; Koutsidis, G.; Lodge, J.; Ashor, A.; Siervo, M.; Lara, J. Tomato and lycopene supplementation and Cardiovasc. risk factors: A systematic review and meta-analysis. Atherosclerosis 2017, 257, 100-108. [CrossRef]

35. Rowles, J.; Ranard, K.; Smith, J.; An, R.; Erdman, J. Increased dietary and circulating lycopene are associated with reduced prostate cancer risk: A systematic review and meta-analysis. Prostate Cancer Prostatic Dis. 2017, 20, 361-377. [CrossRef]

36. Wu, S.; Liu, Y.; Michalek, J.; Mesa, R.; Parma, D.; Rodriguez, R.; Mansour, A.; Svatek, R.; Tucker, T.; Ramirez, A. Carotenoid Intake and Circulating Carotenoids Are Inversely Associated with the Risk of Bladder Cancer: A Dose-Response Meta-analysis. Adv. Nutr. 2019, 11, 630-643. [CrossRef]

37. Nakkeeran, M.; Periasamy, S.; Inmozhi, S.R.; Santha, K.; Sethupathy, S. Increased Levels of Inflammatory Marker hsCRP, MDA and Lipid Profile in Non-obese Hypertension Subjects. Anal. Biochem. 2017, 6, 4.

38. Zhang, Y.; Zhang, J.; Sheng, H.; Li, H.; Wang, R. Acute phase reactant serum amyloid A in inflammation and other diseases. Adv. Clin.Chem. 2019, 90, 25-80. [PubMed]

39. Cumpston, M.; Li, T.; Page, M.; Chandler, J.; Welch, V.; Higgins, J.; Thomas, J. Updated guidance for trusted systematic reviews: A new edition of the Cochrane Handbook for Systematic Reviews of Interventions. Cochrane Database Syst. Rev. 2019, 10, ED000142. [CrossRef]

40. Tacconelli, E. Systematic reviews: CRD's guidance for undertaking reviews in health care. Lancet Infect. Dis. 2010, 10, 226. [CrossRef]

41. Mazidi, M.; Kengne, A.; Katsiki, N.; Mikhailidis, D.; Banach, M. Inverse association between serum antioxidant levels and inflammatory markers is moderated by adiposity: A report based on a large representative population sample of American adults. Br. J. Nutr. 2018, 120, 1272-1278. [CrossRef] [PubMed]

42. Crespo-Sanjuán, J.; Calvo-Nieves, M.; Aguirre-Gervás, B.; Herreros-Rodríguez, J.; Velayos-Jiménez, B.; Castro-Alija, M.; Muñoz-Moreno, M.; Sánchez, D.; Zamora-González, N.; Bajo-Grañeras, R.; et al. Early Detection of High Oxidative Activity in Patients with Adenomatous Intestinal Polyps and Colorectal Adenocarcinoma: Myeloperoxidase and Oxidized Low-Density Lipoprotein in Serum as New Markers of Oxidative Stress in Colorectal Cancer. Lab. Med. 2015, 46, 123-135. [CrossRef] [PubMed]

43. Kim, O.; Yoe, H.; Kim, H.; Park, J.; Kim, J.; Lee, S.; Lee, J.; Lee, K.; Jang, Y.; Lee, J. Independent inverse relationship between serum lycopene concentration and arterial stiffness. Atherosclerosis 2010, 208, 581-586. [CrossRef] [PubMed]

44. Riccioni, G.; D’Orazio, N.; Palumbo, N.; Bucciarelli, V.; di Ilio, E.; Bazzano, L.; Bucciarelli, T. Relationship between plasma antioxidant concentrations and carotid intima-media thickness: The Asymptomatic Carotid Atherosclerotic Disease in Manfredonia Study. Eur. J. Cardiol. Prev. Rehabil. 2009, 16, 351-357. [CrossRef] [PubMed]

45. Hozawa, A.; Jacobs, D.; Steffes, M.; Gross, M.; Steffen, L.; Lee, D. Relationships of Circulating Carotenoid Concentrations with Several Markers of Inflammation, Oxidative Stress, and Endothelial Dysfunction: The Coronary Artery Risk Development in Young Adults (CARDIA)/Young Adult Longitudinal Trends in Antioxidants (YALTA) Study. Clin. Chem. 2007, 53, 447-455.

46. Walston, J.; Xue, Q.; Semba, R.; Ferrucci, L.; Cappola, A.; Ricks, M.; Guralnik, J.; Fried, L. Serum Antioxidants, Inflammation, and Total Mortality in Older Women. Am. J. Epidemiol. 2005, 163, 18-26. [CrossRef]

47. Eboumbou, C.; Steghens, J.; Abdallahi, O.; Mirghani, A.; Gallian, P.; van Kappel, A.; Qurashi, A.; Gharib, B.; De Reggi, M. Circulating markers of oxidative stress and liver fibrosis in Sudanese subjects at risk of schistosomiasis and hepatitis. Acta Trop. 2005, 94, 99-106. [CrossRef]

48. van Herpen-broekmans, W.; Klöpping-ketelaars, I.; Michiel, B.; Cornelis, K.; Hans, P.; Hendriks, F.; Tijburg, L.; van Poppel, G.; Kardinaal, A. Serum carotenoids and vitamins in relation to markers of endothelial. Eur. J. Epidemiol. 2004, 19, 915-921. [CrossRef] 
49. Jonasson, L.; Wikby, A.; Olsson, A. Low serum $\beta$-carotene reflects immune activation in patients with coronary artery disease. Nutr. Metab. Cardiovasc. Dis. 2003, 13, 120-125. [CrossRef]

50. Yadav, D.; Hertan, H.; Schweitzer, P.; Norkus, E.; Pitchumoni, C. Serum and liver micronutrient antioxidants and serum oxidative stress in patients with chronic hepatitis C. Am. J. Gastroenterol. 2002, 97, 2634-2639. [CrossRef]

51. Kritchevsky, S.; Bush, A.; Pahor, M.; Gross, M. Serum Carotenoids and Markers of Inflammation in Nonsmokers. Am. J. Gastroenterol. 2000, 152, 1065-1071.

52. McMillan, D.; Sattar, N.; Talwar, D.; O’Reilly, D.; McArdle, C. Changes in micronutrient concentrations following anti-inflammatory treatment in patients with gastrointestinal cancer. Nutrition 2000, 16, 425-428.

53. Boosalis, M.; Snowdon, D.; Tully, C.; Gross, M. Acute phase response and plasma carotenoid concentrations in older women: Findings from the nun study. Nutrition 1996, 12, 475-478.

54. Almushatat, A.; Talwar, D.; McArdle, P.; Williamson, C.; Sattar, N.; O’Reilly, D.; Underwood, M.; McMillan, D. Vitamin antioxidants, lipid peroxidation and the systemic inflammatory response in patients with prostate cancer. Int. J. Cancer 2005, 118, 1051-1053.

55. McMillan, D.; Talwar, D.; Sattar, N.; Underwood, M.; O’Reilly, D.S.J.; McArdle, C. The relationship between reduced vitamin antioxidant concentrations and the systemic inflammatory response in patients with common solid tumours. Clin. Nutr. 2002, 21, 161-164. [PubMed]

56. Chang, C.; Chen, J.; Ke, D.; Hu, M. Plasma levels of lipophilic antioxidant vitamins in acute ischemic stroke patients: Correlation to inflammation markers and neurological deficits. Nutrition 2005, 21, 987-993. [PubMed]

57. Chung, R.; Leanderson, P.; Lundberg, A.; Jonasson, L. Lutein exerts anti-inflammatory effects in patients with coronary artery disease. Atherosclerosis 2017, 262, 87-93.

58. Quasim, T.; McMillan, D.; Talwar, D.; Sattar, N.; O’Reilly, D.; Kinsella, J. Lower concentrations of carotenoids in the critically ill patient are related to a systemic inflammatory response and increased lipid peroxidation. Clin. Nutr. 2003, 22, 459-462.

59. Nieman, D.; Capps, C.; Capps, C.; Shue, Z.; McBride, J. Effect of 4-Week Ingestion of Tomato-Based Carotenoids on Exercise-Induced Inflammation, Muscle Damage, and Oxidative Stress in Endurance Runners. Int. J. Sport Nutr. Exerc. Metab. 2018, 28, 266-273.

60. Li, Y.; Chang, Y.; Huang, H.; Wu, Y.; Yang, M.; Chao, P. Tomato juice supplementation in young women reduces inflammatory adipokine levels independently of body fat reduction. Nutrition 2015, 31, 691-696.

61. Biddle, M.; Lennie, T.; Bricker, G.; Kopec, R.; Schwartz, S.; Moser, D. Lycopene Dietary Intervention. J. Cardiovasc. Nurs. 2015, 30, 205-212. [PubMed]

62. McEneny, J.; Wade, L.; Young, I.; Masson, L.; Duthie, G.; McGinty, A.; McMaster, C.; Thies, F. Lycopene intervention reduces inflammation and improves HDL functionality in moderately overweight middle-aged individuals. J. Nutr. Biochem. 2013, 24, 163-168. [CrossRef] [PubMed]

63. Petyaev, I.; Dovgalevsky, P.; Klochkov, V.; Chalyk, N.; Pristensky, D.; Chernyshova, M.; Udumyan, R.; Kocharyan, T.; Kyle, N.; Lozbiakova, M.; et al. Effect of lycopene supplementation on Cardiovasc. parameters and markers of inflammation and oxidation in patients with coronary vascular disease. Food Sci. Nutr. 2018, 6, 1770-1777. [PubMed]

64. Gajendragadkar, P.; Hubsch, A.; Mäki-Petäjä, K.; Serg, M.; Wilkinson, I.; Cheriyan, J. Effects of Oral Lycopene Supplementation on Vascular Function in Patients with Cardiovasc. Disease and Healthy Volunteers: A Randomised Controlled Trial. PLoS ONE 2014, 9, e99070.

65. Kim, J.; Paik, J.; Kim, O.; Park, H.; Lee, J.; Jang, Y.; Lee, J. Effects of lycopene supplementation on oxidative stress and markers of endothelial function in healthy men. Atherosclerosis 2011, 215, 189-195. [CrossRef]

66. Markovits, N.; Ben Amotz, A.; Levy, Y. The effect of tomato-derived lycopene on low carotenoids and enhanced systemic inflammation and oxidation in severe obesity. Isr. Med. Assoc. J. 2009, 11, 598-601.

67. Thies, F.; Masson, L.; Rudd, A.; Vaughan, N.; Tsang, C.; Brittenden, J.; Simpson, W.; Duthie, S.; Horgan, G.; Duthie, G. Effect of a tomato-rich diet on markers of Cardiovasc. disease risk in moderately overweight, disease-free, middle-aged adults: A randomized controlled trial. Am. J. Clin. Nutr. 2012, 95, 1013-1022. [CrossRef]

68. Upritchard, J.; Sutherland, W.; Mann, J. Effect of supplementation with tomato juice, vitamin E, and vitamin C on LDL oxidation and products of inflammatory activity in type 2 diabetes. Diabetes Care 2000, 23, 733-738. 
69. Jacob, K.; Periago, M.; Böhm, V.; Berruezo, G. Influence of lycopene and vitamin C from tomato juice on biomarkers of oxidative stress and inflammation. Bri. J. Nutr. 2007, 99, 137-146.

70. Williams, E.; Baines, K.; Smart, J.; Gibson, P.; Wood, L. Rosuvastatin, lycopene and omega-3 fatty acids: A potential treatment for systemic inflammation in COPD; a pilot study. JNIM 2016, 5, 86-95.

71. Rydén, M.; Leanderson, P.; Kastbom, K.; Jonasson, L. Effects of simvastatin on carotenoid status in plasma. Nutr. Metab. Cardiovasc. Dis. 2012, 22, 66-71. [CrossRef] [PubMed]

72. Hurtado-Barroso, S.; Martínez-Huélamo, M.; Rinaldi de Alvarenga, J.; Quifer-Rada, P.; Vallverdú-Queralt, A.; Pérez-Fernández, S.; Lamuela-Raventós, R. Acute Effect of a Single Dose of Tomato Sofrito on Plasmatic Inflammatory Biomarkers in Healthy Men. Nutrients 2019, 11, 851. [CrossRef] [PubMed]

73. Colmán-Martínez, M.; Martínez-Huélamo, M.; Valderas-Martínez, P.; Arranz-Martínez, S.; Almanza-Aguilera, E.; Corella, D.; Estruch, R.; Lamuela-Raventós, R. Trans-Lycopene from tomato juice attenuates inflammatory biomarkers in human plasma samples: An intervention trial. Mol. Nutr. Food Res. 2017, 61, 1600993. [CrossRef]

74. Wood, L.; Garg, M.; Smart, J.; Scott, H.; Barker, D.; Gibson, P. Manipulating antioxidant intake in asthma: A randomized controlled trial. Am. J. Clin. Nutr. 2012, 96, 534-543. [CrossRef]

75. Yeon, J.; Kim, H.; Sung, M. Diets rich in fruits and vegetables suppress blood biomarkers of metabolic stress in overweight women. Prev. Med. 2012, 54, S109-S115. [CrossRef] [PubMed]

76. Dandekar, A.; Mendez, R.; Zhang, K. Cross Talk Between ER Stress, Oxidative Stress, and Inflammation in Health and Disease. Methods Mol. Biol. 2015, 205-214.

77. Furukawa, S.; Fujita, T.; Shimabukuro, M.; Iwaki, M.; Yamada, Y.; Nakajima, Y.; Nakayama, O.; Makishima, M.; Matsuda, M.; Shimomura, I. Increased oxidative stress in obesity and its impact on metabolic syndrome. J. Clin. Investig. 2004, 114, 1752-1761. [CrossRef] [PubMed]

78. Langham, M.; Zhou, Y.; Chirico, E.; Magland, J.; Sehgal, C.; Englund, E.; Mohler, E.; Guo, W.; Barhoum, S.; Wehrli, F. Effects of age and smoking on endothelial function assessed by quantitative Cardiovasc. magnetic resonance in the peripheral and central vasculature. J.Cardiovasc. Magn. Reson. 2015, 17, 1. [CrossRef]

79. Amirkhizi, F.; Siassi, F.; Minaie, S.; Djalali, M.; Rahimi, A.; Chamari, M. Is obesity associated with increased plasma lipid peroxidation and oxidative stress in women? ARYA Atheroscler. 2010, 2, 189-192.

80. Yan, Y.; Guo, T.; Zhu, C. Effects of nonsteroidal anti-inflammatory drugs on serum proinflammatory cytokines in the treatment of ankylosing spondylitis. Biochem. Cell Biol. 2018, 96, 450-456. [CrossRef]

81. Gallelli, L.; Galasso, O.; Falcone, D.; Southworth, S.; Greco, M.; Ventura, V.; Romualdi, P.; Corigliano, A.; Terracciano, R.; Savino, R.; et al. The effects of nonsteroidal anti-inflammatory drugs on Clin.outcomes, synovial fluid cytokine concentration and signal transduction pathways in knee osteoarthritis. A randomized open label trial. Osteoarthr. Cartil. 2013, 21, 1400-1408. [PubMed]

82. Fielding, J.M.; Rowley, K.G.; Cooper, P.; O' Dea, K. Increases in plasma lycopene concentration after consumption of tomatoes cooked with olive oil. Asia Pac. J. Clin. Nutr. 2005, 14, 131-136. [PubMed]

83. Raikos, V. Food matrix: Natural barrier or vehicle for effective delivery of carotenoids from processed foods? Nutr. Metabol. Insights 2017, 1, 1-6.

84. Crowe, K.M. Designing Functional Foods with Bioactive Polyphenols: Highlighting Lessons Learned from Original Plant Matrices. J. Hum. Nutr. Food Sci. 2014, 1, 1018.

85. Krinsky, N.; Yeum, K. Carotenoid-radical interactions. Biochem. Biophys. Res. Commun. 2003, 305, 754-760.

86. Young, A.; Lowe, G. Antioxidant and Prooxidant Properties of Carotenoids. Arch. Biochem. Biophys. 2001, 385, 20-27.

87. Barros, M.; Rodrigo, M.; Zacarias, L. Dietary Carotenoid Roles in Redox Homeostasis and Human Health. J. Agric. Food Chem. 2018, 66, 5733-5740.

88. Niess, A. Response and adaptation of skeletal muscle to exercise-the role of reactive oxygen species. Front. Biosci. 2007, 12, 4826.

89. Halliwell, B. The antioxidant paradox: Less paradoxical now? Br. J. Clin. Pharmacol. 2013, 75, 637-644.

90. Di Pierro, F. Antioxidants and cancer: A debate on prevention, progression, hormesis, and cruciferous vegetables. Nutrafoods 2015, 14, 175-179.

91. Tiwari, A. The antioxidant paradox. Pharmacogn. Mag. 2019, 15, 173. [CrossRef]

92. Biswas, S. Does the Interdependence between Oxidative Stress and Inflammation Explain the Antioxidant Paradox? Oxid. Med. Cell. Longev. 2016, 12, 1-9. [CrossRef] 
93. Bhakkiyalakshmi, E.; Sireesh, D.; Rajaguru, P.; Paulmurugan, R.; Ramkumar, K. The emerging role of redox-sensitive Nrf2-Keap1 pathway in diabetes. Pharmacol. Res. 2015, 91, 104-114. [CrossRef] [PubMed]

94. Lemmens, K.; Sthijns, M.; van der Vijgh, W.; Bast, A.; Haenen, G. The antioxidant flavonoid monoHER provides efficient protection and induces the innate Nrf2 mediated adaptation in endothelial cells subjected to oxidative stress. PharmaNutrition 2014, 2, 69-74. [CrossRef]

95. Bryan, H.; Olayanju, A.; Goldring, C.; Park, B. The Nrf2 cell defence pathway: Keap1-dependent and -independent mechanisms of regulation. Biochem. Pharmacol. 2013, 85, 705-717. [CrossRef] [PubMed]

96. Valgimigli, L.; Iori, R. Antioxidant and pro-oxidant capacities of ITCs. Environ. Mol. Mutagen. 2009, 50, 222-237. [CrossRef]

97. Tanigawa, S.; Fujii, M.; Hou, D. Action of Nrf2 and Keap1 in ARE-mediated NQO1 expression by quercetin. Free Radic. Biol. Med. 2007, 42, 1690-1703. [CrossRef]

98. Jacobs, H.; Moalin, M.; Bast, A.; van der Vijgh, W.; Haenen, G. An Essential Difference between the Flavonoids MonoHER and Quercetin in Their Interplay with the Endogenous Antioxidant Network. PLoS ONE 2010, 5, e13880. [CrossRef]

99. Nespolo, M. Free Radicals in Biology and Medicine; Halliwell, B., Gutteridge, J.M.C., Eds.; University Press: New York, NY, USA, 2007.

100. Kim, Y.; Young, M.; Bobe, G.; Colburn, N.; Milner, J. Bioactive Food Components, Inflammatory Targets, and Cancer Prevention. Cancer Prev. Res. 2009, 2, 200-208. [CrossRef]

Sample Availability: Samples of the compounds are not available from the authors.

(C) 2020 by the authors. Licensee MDPI, Basel, Switzerland. This article is an open access article distributed under the terms and conditions of the Creative Commons Attribution (CC BY) license (http://creativecommons.org/licenses/by/4.0/). 